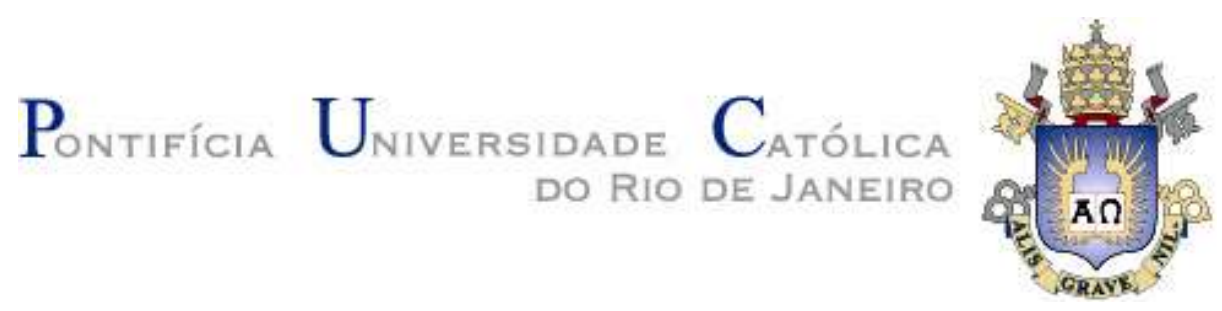

Felipe Araújo Nascimento

\title{
Os Determinantes do Preço de Mercado do Bitcoin
}

Dissertação apresentada ao Programa de Pós-graduação em Administração de Empresas da PUC-Rio como requisito parcial para obtenção do título de Mestre em Administração de Empresas.

Orientador: Prof. Leonardo Lima Gomes

Rio de Janeiro

Abril de 2019 


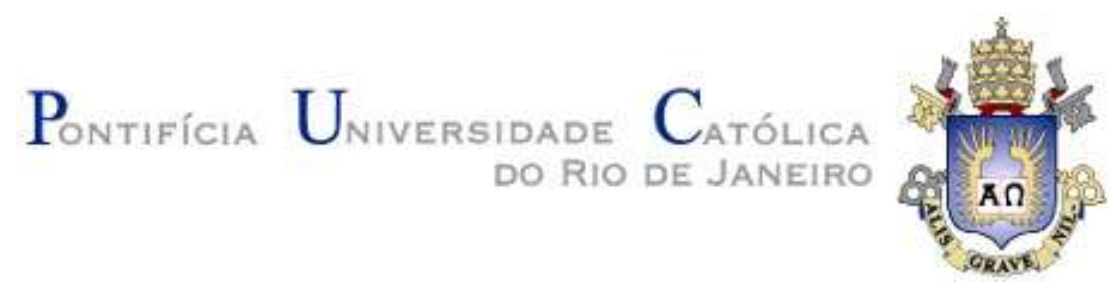

Felipe Araújo Nascimento

\title{
Os Determinantes do Preço de Mercado do Bitcoin
}

Dissertação apresentada como requisito parcial para obtenção do grau de Mestre pelo Programa de Pós-Graduação em Administração de Empresas da PUC-Rio. Aprovada pela Comissão Examinadora abaixo.

\author{
Prof. Leonardo Lima Gomes \\ Orientador \\ Departamento de Administração - PUC-Rio \\ Prof. Henrique Castro Martins \\ Departamento de Administração - PUC-Rio
}

Prof. Carlos Lamare Bastian Pinto

Pesquisador Autônomo

Rio de Janeiro, 15 de Abril de 2019 
Todos os direitos reservados. É proibida a reprodução total ou parcial do trabalho sem autorização da universidade, do autor e do orientador.

\section{Felipe Araújo Nascimento}

Graduou-se em Ciências Econômicas na Universidade Federal do Rio de Janeiro em 2016. Pesquisador das áreas de Finanças Corporativas e Criptomoedas.

Ficha Catalográfica

Nascimento, Felipe Araújo
Os determinantes do preço de mercado do bitcoin /
Felipe Araújo Nascimento ; orientador: Leonardo Lima
Gomes. - 2019.

$61 \mathrm{f}$. : il. color. ; $30 \mathrm{~cm}$

Dissertação (mestrado)-Pontifícia Universidade Católica do Rio de Janeiro, Departamento de Administração, 2019.

Inclui bibliografia

1. Administração - Teses. 2. Bitcoin. 3. Criptomoeda. 4. Moeda digital. 5. Tecnologia. 6. Modelo ADRL. I. Gomes, Leonardo Lima. II. Pontifícia Universidade Católica do Rio de Janeiro. Departamento de Administração. III. Título. 
À minha família e amigos. 


\section{Agradecimentos}

Meu grande agradecimento a minha família pelo imenso apoio nesta trajetoria, os quais me proporcionaram os pilares fundamentais para meu desenvolvimento acadêmico e profissional.

Aos meus amigos e companheiros de mestrado por proporcionarem um grande ambiente para troca de experiências e conhecimentos o qual foi fundamental para a elaboração desse presente trabalho.

Ao meu orientador Professor Leonardo Lima por apresentar e disseminar o conhecimento que foram de pronfunda importância para construção de um trabalho robusto sobre um tema ainda pouco trabalhado no âmbito acadêmico brasileiro.

E a todos os meus demais amigos que contribuíram de forma direta ou indireta para conclusão deste presente trabalho. 


\section{Resumo}

Nascimento, Felipe Araújo; Gomes, Leonardo Lima. Os Determinantes do Preço de Mercado do Bitcoin. Rio de Janeiro, 2019. 61p. Dissertação de Mestrado - Departamento de Administração, Pontifícia Universidade Católica do Rio de Janeiro.

O presente estudo busca entender os principais determinantes da flutuação de preços do Bitcoin através de variáveis relacionadas à força de mercado, tecnologia, reconhecimento público e variáveis macroeconômicas, estimando os coeficientes do vetor de correção de erros (VECM) e do procedimento autoregressivo de defasagens distribuídas (ARDL). Os resultados apresentaram que o reconhecimento público não possui impactos significantes sobre o preço de mercado do Bitcoin, enquanto as forças de mercado, fatores tecnológicos e as variáveis macroêconomicas apresentam impacto significativo em pelo menos um dos modelos utilizados.

\section{Palavras-chave}

Bitcoin; criptomoeda; moeda digital; tecnologia, Modelo ADRL, VECM. 


\section{Abstract}

Nascimento, Felipe Araújo; Gomes, Leonardo Lima (Advisor). The Determinants of the Bitcoin Market Price. Rio de Janeiro, 2019. 61p. MSc. Dissertation - Departamento de Administração, Pontifícia Universidade Católica do Rio de Janeiro.

The present study seeks to understand the main determinants of Bitcoin price fluctuation through variables related to market forces, technology, public recognition and macroeconomic variables, estimating the coefficients of the error correction vector (VECM) and the autoregressive procedure of distributed lags (ARDL). The results showed that public recognition does not have significant impacts on the market price of Bitcoin, while market forces, technological factors and macroeconomic variables have a significant impact on at least one of the models used.

\section{Keywords}

Bitcoin; cryptocurrency; exchange rate; technology; ADRL model; VECM. 


\section{Sumário}

1. Introdução 11

1.1. Problema de Pesquisa 12

1.2. Objetivos 14

1.2.1 Objetivos Específicos $\quad 15$

1.3. Relevância e Justificativa do Estudo 15

1.4. Delimitação do Estudo 16

1.5. Estrutura do Trabalho 16

2. Referencial teórico 17

2.1. O Bitcoin 17

2.1.1 Modus Operandi do Bitcoin 18

2.1.2 Criptografia, Privacidade e Segurança 21

2.1.3 Regulação 22

2.1.4 Vantagens e Desvantagens 23

2.2. Blockchain 25

2.2.1 Funcionamento 25

2.2.2 Demais Aplicações 26

2.2.3 Limitações $\quad 27$

2.3. Blockchains Economics 28

2.3.1 Funções da Moeda 29

2.3.2 Oferta de Bitcoins 30

2.3.3 Demanda por Bitcoins 31

2.3.4 Inflação, Deflação e Política Monetária 32

2.3.5 Determinantes do Preço do Bitcoin 33

3 Metodologia $\quad 35$

3.1 Especificação do Modelo 35

3.2 Variáveis e Dados 36

3.3 Abordagem Econométrica 38

3.3.1 Testes de Raiz Unitária 38

3.3.2 Teste de Cointegração 40

3.3.3 Modelo Vetor de Correção de Erros (VECM) 42 
3.3.4 Procedimento Autoregressivo de Defasagens Distribuídas (ARDL) 43

4 Resultados $\quad 45$

4.1. Análise de Estacionariedade 45

4.2. Análise de Cointegração 49

4.3. Análise do Modelo de Correção de Erros 50

4.4. Análise do Procedimento Autoregressivo de Defasagens Distribuídas

5 Conclusões

6 Referências Bibliográficas

60 
"Investir em conhecimento rende sempre os melhores juros"

Benjamin Franklin 


\section{1 Introdução}

Durante a última década, foi possível observar o surgimento de diversas moedas digitais, as quais cada vez mais vem ganhando relevância e representatividade no cenário economico-financeiro mundial.

A introdução da tecnologia Blockchain, que funciona como um livro-razão mundial, trazendo consigo mais segurança e transparência, permitiu emergir essa nova classe de moedas, lastreada nesse chamado protocolo de confiança (Tapscott, 2016). Baseadas nessa tecnologia, essas novas moedas poderiam ser utilizadas como meio de pagamento em transações cotidianas, com a particularidade de trazer mais segurança e privacidade nas operações, diferentemente das moedas fiduciárias que possuímos hoje. Além disso, não é necessária a figura de um intermediário financeiro nessas transações, ou seja, é possível fazer compras e vendas sem o intermédio de bancos ou qualquer instituição financeira, diferentemente das operações que são feitas com cheques e cartões de crédito nos dias atuais.

O pioneirismo dessas moedas digitais, também rotuladas como criptomoedas ou criptoativos, pertence ao Bitcoin, cuja autoria pertence ao anônimo Satoshi Nakamoto $^{1}$. Apesar do surgimento de seu paper ter acontecido em 2008, a popularização da informação, reconhecimento e de sua utilidade transacional se notabilizou a partir de 2017, quando o Bitcoin começou a ter grande valorização frente ao dólar americano.

${ }^{1}$ Para mais informações: NAKAMOTO, S. Bitcoin: A Peer-to-Peer Electronic Cash System. In: Bitcoin Org, 2008. Disponível em:https://bitcoin.org/bitcoin.pdf. 


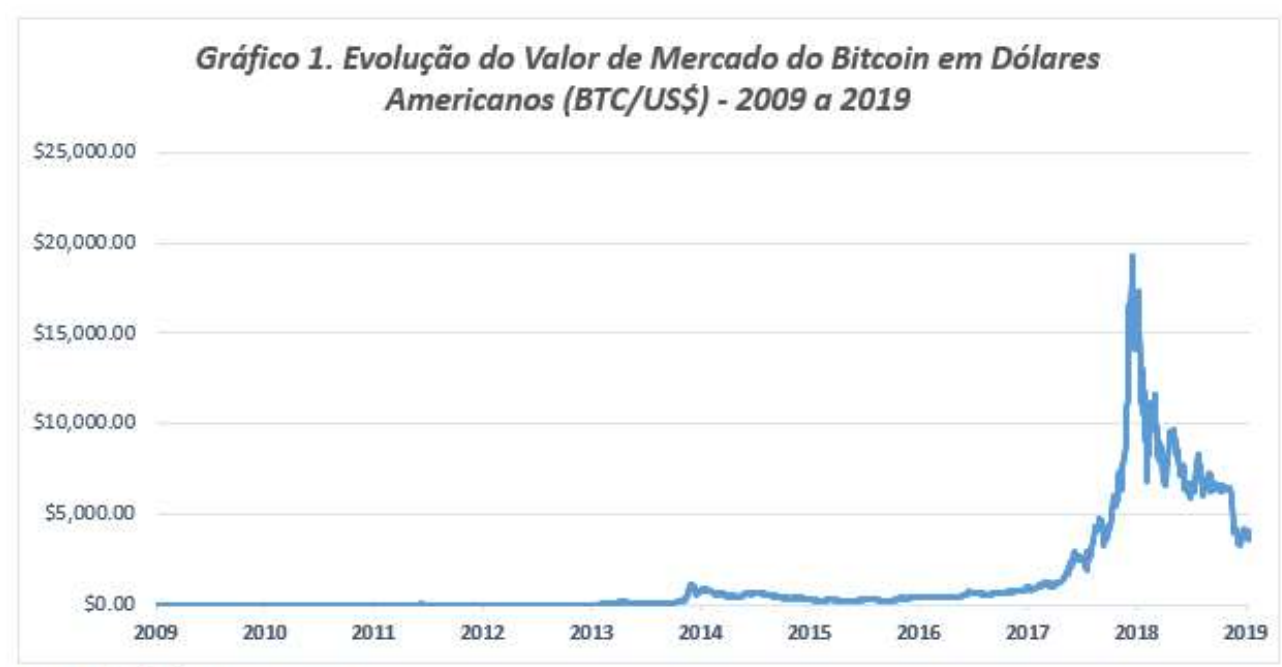

Fonte: Blockchain.com, 2019

No final 2017, o Bitcoin alcançou seu maior valor de mercado, aproximadamente US\$20.000,00, sendo o maior valor registrado para uma criptomoeda até o desenvolvimento desse trabalho.

Com o surgimento do Bitcoin, foi possível observar, a seguir, a criação de um mercado para criptomoedas, surgindo uma infinidade de alternativas ao Bitcoin, como o Litecoin e o Ethereum - criptomoedas com suas particularidades, mas que continuam sendo altamente intensivas em tecnologia, cada vez mais gerando disrupção no cenário econômico-financeiro contemporâneo.

\section{1.}

Problema de Pesquisa

Observando o grande movimento de preços do Bitcoin durante os últimos anos, além de sua notória popularização, pesquisadores buscam explicar quais são os determinantes do preço dessa criptomoeda, a fim de explicar a grande volatilidade de sua série de preço.

Buchholtz et al. (2012) definiram e utilizaram a função de oferta e demanda por Bitcoin para entender a volatilidade dos preços da criptomoeda. Em seu paper, definiam a oferta de Bitcoin basicamente em função da quantidade de Bitcoins em 
circulação, a qual é exogenamente definida, enquanto a função de demanda é determinada pelas transações efetuadas como meie pagamento de bens e serviços. Entre os resultados, foram encontrados indícios de comportamento especulativo e que poderia existir uma bolha no mercado de Bitcoins, sendo analizado o período de 2010 a 2012.

Kristoufek (2013) argumentou que o comportamento da série de preços do Bitcoin não poderia ser explicado de forma satisfatória por teorias econômicas e financeiras, tais como modelos de fluxo de caixa futuros, paridade do poder de compra ou paridade de taxa de juros. $\mathrm{O}$ autor se estende afirmando que não há fundamento macroeconônico para moedas digitais e defende que a emoção do investidor é uma variável crucial para entender as flutuações. Utilizando as ferramentas de busca Wikipedia e Google como proxy para emoção do investidor, é encontrada uma correlação positiva entre o preço do Bitcoin e as proxies.

Outros autores corroboram com a ideia de não limitar o modelo somente em variáveis macroeconômicas a fim de encontrar os determinantes do Bitcoin, como Bouoiyour e Selmi (2015). Os resultados desse estudo para o período de 2010 a 2014 revelaram um comportamento altamente especulativo do Bitcoin e coeficientes significantes para o impacto positivo das variáveis atratividade para os investidores e o índice de mercado de Shangai, que é um grande mercado para a economia do Bitcoin.

A abordagem de van Wijk (2013) buscou entender o valor de mercado do Bitcoin através do índice de ações de diversos mercados, taxas de câmbio e preço do petróleo. Seus resultados apresentaram que o índice Dow Jones, a taxa de câmbio euro-dólar e os preços do petróleo têm impacto significante no preço do Bitcoin no longo prazo.

Outros autores, como Lin e Wang (2016), buscam adicionar fatores tecnológicos intrínsecos ao Bitcoin, além de fatores econômicos, a fim de entender a formação de preço da criptomoeda. $\mathrm{Na}$ análise desses autores, sugere-se que fatores tecnológicos tem um importante papel na determinação do preço do Bitcoin em termos de tecnologia de mineração e reconhecimento público. Entre seus resultados, foi possível identificar um impacto significativo positivo sobre o preço da variável tecnologia de mineração e um impacto negativo da dificuldade de mineração sobre o preço do Bitcoin. 
Em um trabalho recente, Ciaian, Rajcaniova e Kancs (2016) buscaram utilizar um framework clássico utilizando as forças de mercado, oferta e demanda, e adicionaram variáveis de forma a mensurar a atratividade da criptomoeda para investidores e para usuários. Em seus resultados, confirmaram que as forças de mercado têm importante papel na formação do preço do Bitcoin, que novas informações sobre a moeda não têm impacto no longo prazo e que não é possível rejeitar a hipótese de a especulação do investidor ter impacto na formação do preço do Bitcoin.

Em síntese, existem diversas abordagens utilizadas no cenário acadêmico, as quais buscam examinar as variáveis que possuem impacto, direto ou indireto, sobre os preços da série do Bitcoin, partindo de premissas econômicas básicas, como as funções de oferta e demanda pela criptomoeda, até variáveis ligadas à tecnologia e desempenho da moeda virtual, além de fatores psicológicos de usuários e investidores.

Não obstante, o presente estudo busca apresentar fatores econômicos e tecnológicos que facilitem as interpretações das flutuações de preço do Bitcoin, analisando o período de 2017 a 2018, no qual ocorrem as principais e mais abruptas variações no preço. De forma mais específica, o presente trabalho analisará a interação do valor de mercado do Bitcoin com variáveis que correspondem a fatores tecnológicos e de reconhecimento público, de fatores de mercado relacionados à moeda e a um grupo de índice de ações de alguns mercados, sendo estudado através de 2 modelos: Vetor de Correção de Erros (VECM) e procedimento Autoregressivo de Defasagens Distribuídas (ARDL).

\section{2 .}

\section{Objetivos}

Apresentar as principais variáveis que possuem papel importante na determinação do preço de mercado do Bitcoin, buscando entender as interações desse novo ativo com variáveis macroeconômicas, financeiras e tecnológicas.

O presente estudo é fundamentado em uma pesquisa quantitativa, cuja fonte de dados é estritamente secundária, utilizando dados de diversos sites com reputação de significativa relevância. 


\subsection{1}

Objetivos Específicos

Para o cumprimento do objetivo expresso acima, são previstos objetivos específicos, os quais também buscarão ser respondidos, tais como:

- Verificar a existência de interação entre a série de preço do Bitcoin e variáveis relacionadas a fatores tecnológicos da criptomoeda;

- Verificar a existência de interação entre a série de preço do Bitcoin e variáveis relacionadas às forças de mercado, tais como a oferta e demanda por Bitcoin;

- Verificar a existência de interação entre a série de preço de Bitcoin e variáveis do cenário macroeconômico e financeiro mundial, tais como índices de ações e taxas de juros;

- Verificar a existência de interação entre a série de preços do Bitcoin e o reconhecimento público dos usuários e investidores.

\section{3.}

Relevância e Justificativa do Estudo

A pesquisa se propõe a estudar um tema que ainda carece na literatura nacional e internacional e que vem ganhando espaço no sistema econômicofinanceiro mundial. O estudo econômico por trás de criptomoedas, como o Bitcoin, ainda está em sua fase inicial e, apesar de nenhum estudo ter se notabilizado, as discussões acerca dessa temática geram interesse de uma série de indivíduos buscando entender qual é o papel dessa nova gama de moedas nos rumos da economia das nações.

Esse estudo tende a somar a alguns artigos que buscam entender a formação de preço do Bitcoin, se notabilizando por estudar os anos mais recentes da série de preços (2017 - 2018), onde se encontram grandes flutuações, e também reconhecido como o período de maior popularização desse tema. A literatura encontrada acerca desse tema se limita a estudar a série até o ano de 2016. 
A intenção do presente estudo é apresentar, a partir de uma ótica econométrica, as variáveis que interagem com o Bitcoin a fim de explorar a microeconomia e a macroeconomia de criptomoedas, contribuindo para a proliferação e disseminação do conhecimento sobre essa nova classe de ativos.

\section{4.}

Delimitação do Estudo

O presente estudo buscará apontar e entender os determinantes da formação de preço do Bitcoin, aplicando conceitos da macroeconomia, microeconomia e de finanças corporativas, a fim de respaldar na teoria as variáveis que interagem com o preço dessa criptomoeda. Para isso, também será utilizado o ferramental econométrico, utilizando-se dois modelos amplamente conhecidos, buscando reforçar os achados desse estudo.

No entanto, esse estudo não tem intenção de valorar o Bitcoin como um ativo financeiro, buscando encontrar qual seria seu preço justo. Além disso, não há intenção de explorar o valor intrínseco da Blockchain - tecnologia base do Bitcoin e que está presente em diversas classes de criptomoedas. Esses temas possuem grande valor acadêmico e profissional, no entanto, não serão abordados nesse trabalho, assim podendo ser explorados em próximos estudos.

\section{5 .}

\section{Estrutura do Trabalho}

O presente trabalho está estruturado em cinco capítulos, de modo que esta introdução corresponde ao capítulo inicial, fundamentando o problema de pesquisa, os objetivos, a relevância e a delimitação do estudo. O segundo capítulo aborda o referencial teórico da variável dependente e das variáveis independentes do modelo econométrico. $\mathrm{O}$ terceiro capítulo apresenta a abordagem metodológica utilizada para calcular e interpretar os resultados. $\mathrm{O}$ quarto capítulo corresponde à análise dos resultados e interpretações do modelo e, por fim, o último capítulo apresenta as conclusões e comentários. 


\section{2. Referencial teórico}

Este capítulo tem por objetivo estabelecer a base teórica a qual será utilizada para fundamentar a construção de um modelo preditivo para o valor de mercado do Bitcoin, assim como as ferramentas e a base conceitual para análise e interpretação dos resultados. O desenvolvimento desse arcabouço teórico foi realizado a partir da revisão da recente literatura de criptomoedas e sua arquitetura econômica, além de apresentar a formulação das hipóteses de fatores os quais influenciam na formação de preço da criptomoeda. A construção da revisão dessa literatura reflete o esforço do pesquisador em ampliar os estudos na área de formação de preço de criptomoedas, apoiando-se em resultados de pesquisas anteriores.

\section{1. \\ O Bitcoin}

O Bitcoin surgiu em 2008 após a publicação do paper "Bitcoin: A peer-topeer Electronic Cash System", cuja autoria é dada a Satoshi Nakamoto, um pseudônimo para um indivíduo ou um grupo de indivíduos os quais ainda não tiveram suas verdadeiras identidades publicadas.

Em seu paper original, essa criptomoeda se apresenta como uma moeda virtual par a par (ou ponto a ponto), a qual permite pagamentos online sem a dependência de um intermediário, como instituições financeiras ou governos (Nakamoto, 2008).

O ideal de criação de um meio de troca que não tivesse interferência de um terceiro, seja uma instituição financeira ou instituições governamentais, se iniciou com Wei Dai e seu protocolo que fundamentava o B-Money, em 1998, no qual apresentava as principais características de uma moeda digital e quais poderiam ser suas aplicações práticas - muitos desses princípios os quais foram utilizados para fundamentar a criação do protocolo Bitcoin. Em seu artigo, Wei Dai (1998, p. 1) 
apresentava um viés político e econômico para criação de seu protocolo, onde argumentava que

Até agora não está claro, mesmo teoricamente, como tal comunidade poderia operar. Uma comunidade é definida pela cooperação de seus participantes e uma cooperação eficiente requer um meio de troca (dinheiro) e uma maneira de fazer cumprir os contratos. Tradicionalmente, esses serviços tem sido fornecidos pelo governo ou instituições patrocinadas pelo governo e apenas para pessoas jurídicas [...] Eu descrevo um protocolo pelo qual esses serviços podem ser fornecidos para e por entidades não rastreáveis.

Diferentemente do artigo com viés político de Wei Dai (1998), o paper de Satoshi Nakamoto (2008) foi estruturado de uma forma conceitual e prática sobre seu objeto de estudo, não apresentando explicitamente qualquer viés ideológico em seu texto. No entanto, seu pensamento político-filosófico foi explicitado em uma lista de discussões pública onde respondia perguntas de seus participantes, conforme identificado por Fernando Ulrich (2016, p. 42), e que corroboram com o pensamento anárquico apresentado por Wei Dai (1998). O viés político-fillosófico na qual foi baseada a criação do Bitcoin não é cerne deste trabalho, mas nos auxilia na intuição e na interpretação do protocolo Bitcoin.

\subsection{1 \\ Modus Operandi do Bitcoin}

Sempre que discutimos sobre transações comerciais, principalmente no século XXI, estamos, na maioria das vezes, utilizando um intermediário para realizar as transações, excluindo basicamente o cenário onde é utilizado o dinheiro físico, na maioria dos casos papel moeda. Nakamoto $(2008$, p. 1) argumenta que a mediação feita por intermediários aumenta os custos de transação, limitando o tamanho da transação que pode ser realizada e excluindo a possibilidade de pequenas transações casuais. Acrescenta, ainda, que "uma certa percentagem de fraude é aceita como inevitável" (Nakamoto, 2008, p. 1).

O funcionamento do Bitcoin aparece como solução a esses tipos de problemas, apresentando um sistema de pagamentos digitais, o qual se fundamenta em criptografia e proof-of-work (usualmente traduzido como prova de esforço), temas que serão retratados durante esse capítulo. Esses mecanismos minimizam o acontecimento de fraudes e diminuem os custos de transação, trazendo consigo 
mais segurança em uma transação e permitindo ilimitadas formas de transações que anteriormente não se apresentavam viáveis.

Nakamoto $(2008$, p. 2) começa definindo em seu paper uma moeda eletrônica como uma cadeia de assinaturas digitais, de forma que cada proprietário pode transferir essa moeda pela assinatura digital de um hash (algoritmo do Bitcoin) de uma transação anterior e uma chave pública do novo proprietário. Em outras palavras, os usuários do Bitcoin possuem duas chaves, uma pública e uma privada. A chave pública será utilizada no momento da transação e, assim que a transação for autenticada, passará a compor a cadeia de dados do Bitcoin, que passaremos a chamar mais tarde de Blockchain. Toda a transação na rede Bitcoin se torna pública e imutável.

Um grande problema resolvido pelo protocolo Bitcoin foi a questão do gasto duplo (ou double-spending), que consiste basicamente em utilizar o mesmo dinheiro em mais de uma transação pela mesma pessoa. Sem intermediários, não haveria alguém que armazenasse o registro do histórico de transações e o saldo contábil de um indivíduo a fim de garantir fundos para a realização de uma transação. Fernando Ulrich (2016, p. 16) fez uma analogia desse problema utilizando a mensagem de um e-mail, que simplifica e sumariza a explicação desse problema:

Imagine que não haja intermediários com registros históricos, e que o dinheiro digital seja simplesmente um arquivo de computador, da mesma forma que documentos digitais são arquivos de computador. Maria poderia enviar para João 100 unidades monetárias simplesmente anexando o arquivo de dinheiro em uma mensagem. Mas assim como ocorre com o e-mail, enviar o arquivo como anexo não o remove do computador originador da mensagem eletrônica, [...].

A solução foi a implementação de um servidor timestamp distribuído em uma base par a par (ou ponto a ponto), aplicando um sistema de proof-of-work (prova de esforço ou prova do trabalho) para validação de transações. Essa solução consistia basicamente em um servidor descentralizado onde é feito o registro e a autenticação de todas as transações na rede Bitcoin. O registro, a autenticação e a conciliação de uma transação são feitos computacionalmente pelos chamados "mineradores", os quais tem papel fundamental no processo, garantindo a veracidade de uma transação realizada dentro da rede. Os incentivos a esses mineradores que suportam a rede são basicamente dois: a remuneração em Bitcoins recém-criados e uma taxa 
de transação. Segundo Nakamoto (2008, p. 4), "os incentivos dados aos mineradores podem encorajá-los a continuar honestos”.

De forma resumida, Satoshi Nakamoto (2008, p. 6) apresenta o funcionamento da rede Bitcoin em seis passos, conforme a seguir: 1. Novas transações são transmitidas para todos os nodes (ou participantes da rede); 2. Cada node agrupa uma nova transação a um bloco; 3 . Cada node trabalha para encontrar a proof-of-work (prova de esforço) para aquele bloco; 4. Quando o node encontra a prova de esforço, o bloco é transmitido para todos os nodes; 5. Nodes aceitam o bloco somente se todas as transações contidas no bloco são válidas e não previamente despendidas; 6. Nodes expressam a aceitação do bloco, criando o próximo bloco da cadeia, usando o hash (protocolo do Bitcoin) do bloco aceito como hash prévio do próximo bloco.

Crosby et al. (2016) apresentam em seu paper um esquema a fim de ilustrar e simplificar o funcionamento de uma transação de Bitcoin na rede Blockchain, conforme abaixo:

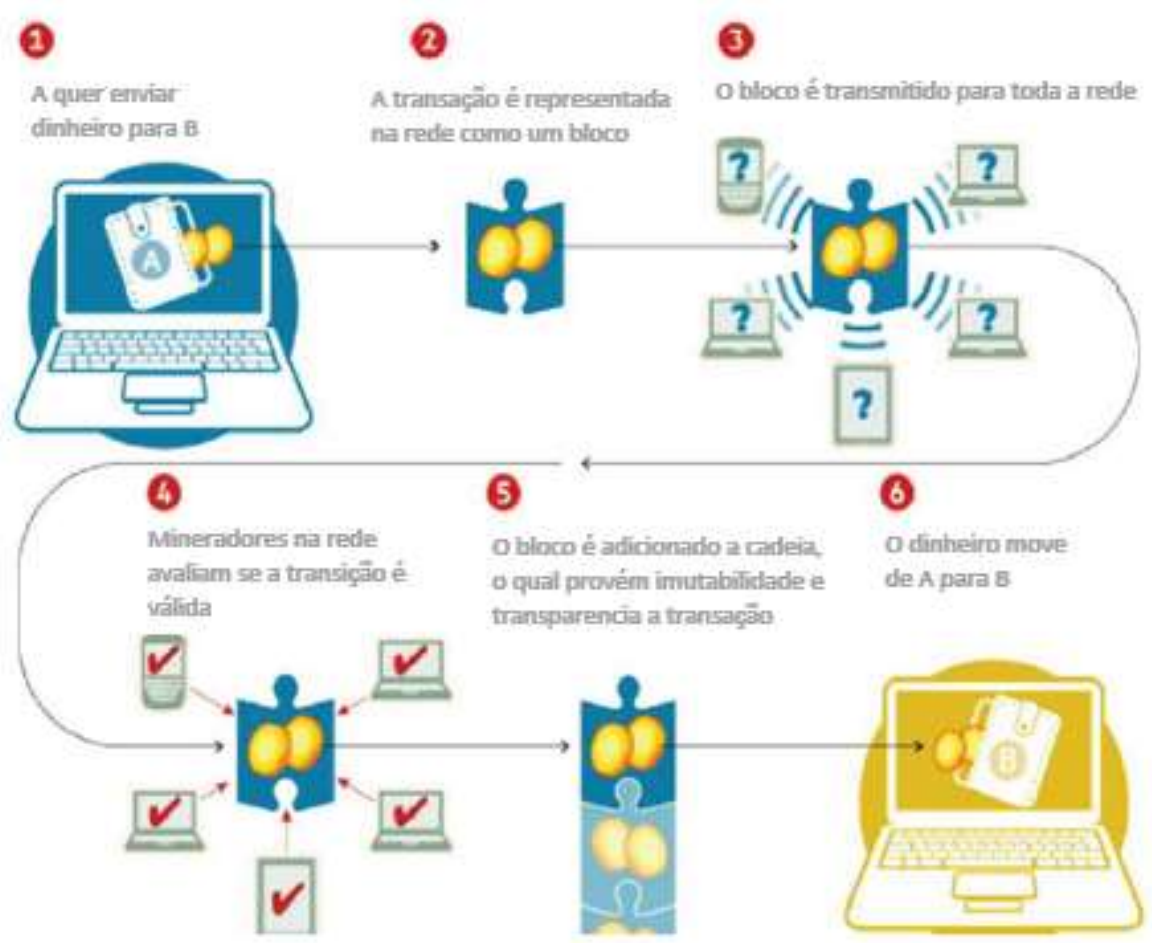

Figura 1. Transaçẫo utilizando a rede Bitcoin.

Fonte: Crosbyet al. (2016), adaptado pelo autor. 


\subsection{2}

\section{Criptografia, Privacidade e Segurança}

Duas questões que tangem a discussão de criptomoedas são relacionadas à privacidade e segurança das transações pela internet, onde o Bitcoin torna-se alvo de especulações e críticas, pois é a criptomoeda pioneira e de maior capitalização de mercado.

O modelo tradicional utilizado por instituições financeiras mantém um certo nível de privacidade, limitando o acesso à informação entre as partes envolvidas e o intermediário (Nakamoto, 2009, p. 6). O Bitcoin funciona de forma completamente diferente, utilizando o conceito de chaves públicas e privadas (Albuquerque; Callado, 2016, p. 10). A chave pública, nesse contexto, é utilizada para efetuar qualquer transação na rede e é simultaneamente registrada na cadeia de blocos do Bitcoin, tornando-se pública. No entanto, não é uma tarefa simples atrelar a chave pública de um participante da rede à sua identidade (ou chave privada). Nakamoto $(2009$, p. 6) faz uma analogia desse processo ao mercado de ações, onde o nível de informação pública se limita ao tamanho e ao tempo de transação.

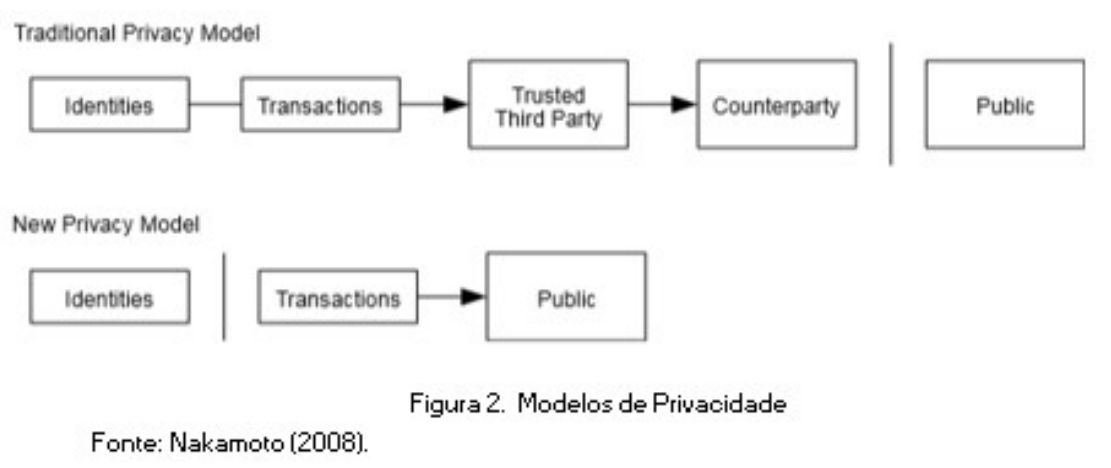

Fernando Ulrich (2016, p. 21) argumentou que o Bitcoin se encontra em um ponto entre uma transação entre dinheiro vivo e a transação realizada por um intermediário, no sentido de que, quando a transação é realizada em dinheiro vivo, não existe a presença de um intermediário financeiro que conheça suas respectivas identidades, mas, por outro lado, as transações realizadas na rede são registradas e armazenadas, da mesma forma que um intermediário faz com seus clientes, com a grande diferença de que as informações são públicas. 
Com relação a ataques cibernéticos que possam criar novos Bitcoins de maneira ilícita, a criptografia do protocolo Bitcoin é uma forma robusta de assegurar que tais ações sejam improváveis. Albuquerque e Callado (2016, p. 9) comentam sobre a regra de criação de um hash, necessário para criação de novos Bitcoins, os quais precisam possuir um certo número de consecutivos zeros em seu código, sendo que a atual quantidade de zeros determina a dificuldade de criação de novos blocos - quanto maior o número de zeros consecutivos necessários, mais difícil será encontrar um resultado válido. A dificuldade é automaticamente ajustada pela rede, de acordo com a taxa de mineração ${ }^{2}$. A criptografia e seus respectivos códigos não são temas centrais desse estudo, mas ajudam a dimensionar a complexidade da segurança da criptomoeda.

Apesar da robustez e complexidade do protocolo Bitcoin, diversas críticas sobre a segurança provida pela rede são minadas por ataques bem-sucedidos de hackers, principalmente de casas de câmbio de Bitcoin. Em sua obra, Fernando Ulrich (2016) enumera alguns ataques, com destaque a Mt.Gox, a casa de câmbio de Bitcoins mais popular em 2013. Ainda argumenta que “[...] muitos dos riscos de segurança enfrentados pelo Bitcoin são similares àqueles com os quais moedas tradicionais também se defrontam" (Ulrich, 2016, p. 30). Os Bitcoins, como qualquer moeda, podem ser roubados, visto a violação de informações financeiras, como acontecem em bancos. Nesses casos, os usuários precisam tomar precauções que assegurem que tais informações não sejam violadas.

\subsection{3 \\ Regulação}

Outro tópico polêmico quando se trata de Bitcoin é quanto a sua regulação. Apesar de já possuir dez anos desde sua criação, tal questão ainda é uma zona cinzenta no cenário mundial. A criação de uma regulação para tal classe de ativos não é prevista pelo autor, Satoshi Nakamoto (2008), ainda mais quando consideramos seu viés político e ideológico que tange ao anarquismo. No entanto, diversos Estados buscam entender e encontrar uma solução para o papel regulatório

\footnotetext{
${ }^{2}$ Essa taxa é definida por quantos Bitcoins serão minerados em um certo período de tempo.
} 
de criptomoedas, como o Bitcoin, visto a utilização ilícita desse ativo para atividade de evasão de divisas, lavagem de dinheiro e comercialização de produtos ilícitos.

Albuquerque e Callado (2016) apresentam dois casos onde governos tomaram posições quanto a utilização e tributação do Bitcoin dentro de suas divisas. $\mathrm{O}$ primeiro caso se refere a Singapura, primeiro Estado que publicou orientações de como os Bitcoins deveriam ser taxados. Uma de suas orientações é que

empresas que compram e vendem Bitcoins devem ser taxadas sobre o ganho de suas vendas de Bitcoins, mas, por outro lado, se os Bitcoins fizerem parte do portfólio de investimento de uma companhia adquiridas para propósitos de investimento de longo prazo, os ganhos da venda serão considerados capital em natureza, assim não serão taxados (Farr, 2014) ${ }^{3}$.

O segundo caso tange à Tailândia, a qual baniu o uso do Bitcoin dentro de todo seu território. Melanie Swan (2015) apresenta Bangladesh, Bolívia, Equador, Islândia, Quirquistão e Vietnã como países em que o Bitcoin também foi banido.

\section{1 .4 Vantagens e Desvantagens}

É sempre destacado que uma das principais vantagens do Bitcoin é a diminuição dos custos de transação. Nakamoto (2008) já destacava em seu paper que sua criação iria reduzir os custos de transação, permitindo a realização de transações que antes não eram permitidas com os altos custos no modelo com a presença de um intermediário. Pois bem, a ausência de um intermediário na rede Bitcoin, como instituições financeiras e governos, reduzem significantemente custos os quais estão presentes na maioria das transações realizadas com cartões de crédito e cheques, ou até mesmo ao se manter uma conta em um banco ativa. Isso é permitido pelo modelo descentrado do Bitcoin, ou peer-to-peer (conhecido como ponto a ponto ou apenas P2P). A utilização dessa criptomoeda permite que pequenos negócios tenham acesso a um meio de pagamento eficiente sem as altas cobranças de taxas de cartão de crédito feita por instituições financeiras. Além disso, a utilização do Bitcoin minimiza o risco de fraudes, visto que todas as

\footnotetext{
${ }^{3}$ Para informações adicionais sobre o tema: https://venturebeat.com/2014/01/10/singapore-clarifies-tax-on-bitcoin-exchanges-andsales/
} 
transações são armazenadas na cadeia de dados da criptomoeda, onde as informações são públicas e imutáveis.

Outro ponto que favorece o uso de Bitcoins é a promessa de remessa de divisas ao exterior a baixo custo, cujas taxas implícitas são menores que as taxas dos atuais bancos que prestam esse serviço, além de serem mais ágeis na transferência de recursos.

O Bitcoin ainda pode ser utilizado no combate à desigualdade social, no sentido de que provém acesso a recursos financeiros baratos e que, por muitas vezes, não se apresentam disponíveis para populações de mais baixa renda, principalmente em países subdesenvolvidos. Esse criptoativo também pode ser utilizado no combate à opressão das minorias, garantindo o anonimato das transações, como destacado por Fernando Ulrich (2016).

No entanto, essa inovação tecnológica e financeira não está blindada de desvantagens. As flutuações do preço de mercado do Bitcoin são enormes, basta recorrer ao Gráfico 1, onde o preço do Bitcoin ainda não foi estabilizado e ainda está exposto a diversas bolhas especulativas de investidores, como constatado nos achados de Buchholtz et al. (2012) e Bouoiyour e Selmi (2015).

Outro ponto de atenção sobre o Bitcoin se refere às questões de violações de segurança às quais os usuários podem ficar expostos, de maneira que o roubo de informações financeiras pessoais podem levar à perda da propriedade dos Bitcoins, como comentado durante esse capítulo, onde algumas casas de câmbio de Bitcoin perderam seus criptoativos devido a esses tipos de violações.

Por fim, esse criptoativo vem sendo utilizado para fins criminosos a fim de ajudar na lavagem de dinheiro, evasão de divisas e comercialização de mercadorias ilegais, visto a privacidade que o Bitcoin provém aos seus usuários. Um caso recorrente na literatura, citado por Fernando Ulrich (2016) e Albuquerque e Callado (2016), é o caso da Silk Road, um site do mercado negro, onde são comercializados diversos produtos, lícitos e ilícitos, por meio do Bitcoin.

Em suma, a utilização do Bitcoin deve ser utilizada com sabedoria, pois possui muitas fortalezas, mas também pontos frágeis, os quais podem ser utilizados para atividades oportunistas. 


\section{2. \\ Blockchain}

Evitamos durante a seção sobre o Bitcoin utilizar a nomenclatura da Blockchain. No entanto, o conceito desse termo esteve presente em toda a descrição sobre a criptomoeda e merece uma seção a parte para explicar o que de fato é a Blockchain, suas funcionalidades e sua importância para o funcionamento do Bitcoin.

Don e Alex Tapscott (2016, p. 36) definem a Blockchain como um livro-razão (ledger), distribuído globalmente, e que possui como alicerce fundamental um "protocolo de confiança" - o qual pode ser interpretado como um protocolo que permite que pessoas criem confiança através de um código inteligente. Os autores fazem um contraponto com as habituais transações dizendo que

transações confiáveis diametralmente entre duas ou mais partes, autenticadas pela colaboração em massa e alimentada por interesses coletivos, em vez de grandes corporações motivadas pelo lucro (Tapscott; Tapscott, 2016, p. 35).

Melanie Swan (2015, p. X) corrobora com essa definição, mas acrescenta em sua definição a figura do Bitcoin, dizendo que o Blockchain é um livro-razão público, onde todas as transações de Bitcoins são realizadas. Destaca-se que é a principal inovação tecnológica do Bitcoin e que suas aplicações não se limitam ao campo de moedas.

\subsection{1}

\section{Funcionamento}

É simples entender o funcionamento de uma Blochchain após ter definido o modus operandi do Bitcoin. A Blockchain funciona basicamente como um livrorazão público onde são armazenadas todas as transações efetuadas. No caso do Bitcoin, o tempo para adição de um bloco à cadeia é de 10 minutos (Swan, 2015, p. $\mathrm{X})$.

Don e Alex Tapscott (2016, p. 37), destacam três grandes características do Blockchain: distribuído, público e criptografado. Ele é distribuído no sentido em que não possui um servidor central, mas sim é distribuído entre todos os participantes da rede ao redor do mundo. Essa característica foi o que chamamos de 
peer-to-peer (ponto a ponto ou $\mathrm{P} 2 \mathrm{P}$ ) quando falávamos sobre o funcionamento do Bitcoin. A segunda característica é sua publicidade, qualquer indivíduo pode consultar todas as transações incorridas dentro da rede Blockchain, não possuindo um intermediário que prive essa informação de toda a população. Por fim, sua rede é altamente criptografada, possuindo uma chave pública e privada, a fim de garantir a segurança do usuário na rede.

Melanie Swan (2015) apresenta uma abordagem interessante que nos ajuda a entender o papel do Blockchain no funcionamento do Bitcoin. Ela denota o Bitcoin como três diferentes temas, o primeiro se refere à plataforma tecnológica da Blockchain, a rede descentralizada onde são encontradas todas as transações realizadas em Bitcoins. O segundo tema é referido como seu próprio protocolo, o sistema utilizado para transferir a moeda através da Blockchain. E o terceiro é o papel de moeda digital, que funciona como meio de troca.

\subsection{2}

\section{Demais Aplicações}

A Blockchain não limita suas aplicações no campo da moeda. Seu uso mais atual tem sido em torno da discussão de contratos inteligentes. Swan (2015) se refere ao atual momento como Blockchain 2.0, onde é utilizado para a descentralização de mercados, os quais contemplam a transferência de muitos tipos de ativos. A Blockchain 1.0, nesse caso, se refere à descentralização da moeda e dos pagamentos, disrupção que podemos observar com o Bitcoin.

Em suma, a utilização da Blockchain é sinérgica na economia de contratos, onde pode-se utilizar a rede para registrar, confirmar e transferir toda a espécie de contratos e propriedade. A Tabela 1 apresenta alguns exemplos de aplicações da Blockchain em outros mercados. 


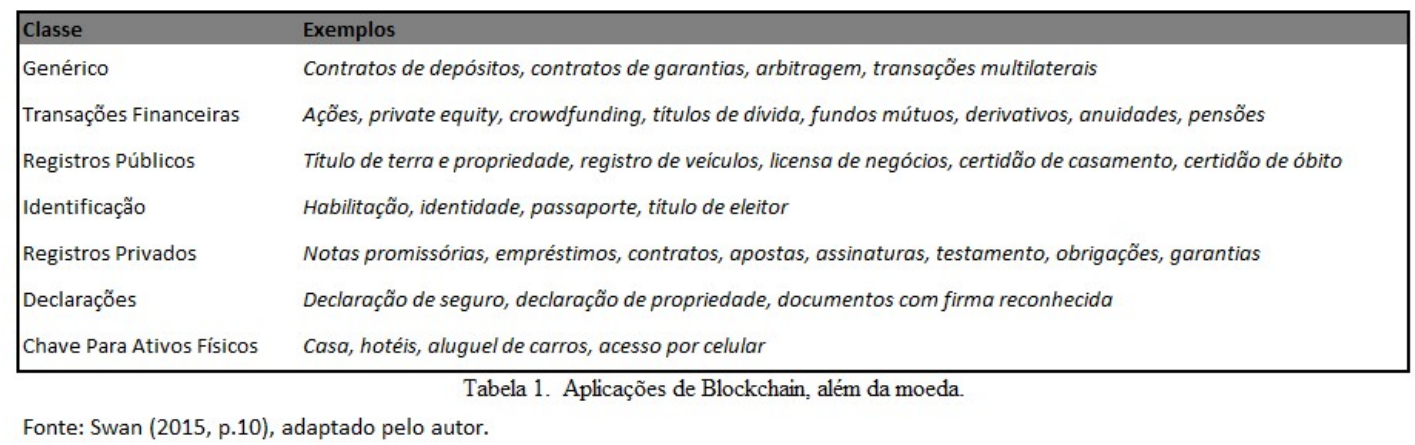

Outra aplicação interessante no que tange à Blockchain se refere aos Prediction Markets, que funcionam como um local de apostas para os resultados do mundo real, como eleições, legislações, partidas esportivas e lançamento de novos produtos, além de ser uma boa fonte de informação para o desenvolvimento da indústria Blockchain (Swan, 2015, p. 13). Prediction Markets são uma maneira de observar o que os insiders acham sobre a direção futura dos preços do Bitcoin, por exemplo.

\subsection{3 Limitações}

São notáveis as inúmeras vantagens que a Blockchain apresenta, criando uma grande disrupção, no início do século XXI, e abrindo uma gama de oportunidades para inovações. No entanto, também há notáveis limitações atreladas ao Blockchain que precisam ser superadas para que a sua utilização seja popularizada.

Swan (2015) apresenta em sua obra um grande desafio a ser superado, que consiste na atual taxa de transmissão da rede, apresentando o exemplo do Bitcoin. Atualmente, a tecnologia que lastreia o Bitcoin permite somente o total de 7 transações por segundo. Em contrapartida, outras redes de transmissões, como a VISA, chegam a processar um número de 10 mil transações por segundo em seu pico.

Outro grande problema está em torno da latência da rede. Atualmente, uma transação de Bitcoin no Blockchain possui tempo estimado de 10 minutos, isso significa que é necessário aguardar 10 minutos até a transação ser confirmada. Caso o tamanho da transação cresça significativamente, é necessário mais tempo para a 
transação ser autenticada, diferentemente da VISA, que leva somente segundos (Swan, 2015, p. 82).

Tamanho e a largura da banda é uma outra limitação notável e que se relaciona com as demais limitações citadas. O tamanho da Blockchain hoje, por exemplo, supera os 25 GB (Swan, 2015, p. 82). Apesar de não ser um tamanho considerável hoje, visto a capacidade de armazenamento padrão dos computadores atuais, caso a questão da taxa de transmissão e da latência for resolvida, o tamanho do Blockchain tende a aumentar consideravelmente, aumentando o tempo de download de todo ecossistema da Blockchain no computador de um indivíduo lembrando que esse sistema consiste em uma rede peer-to-peer, ou seja, descentralizada.

Outra grande polêmica em torno da Blockchain consiste no consumo de energia necessário à mineração. Swan $(2015$, p. 83) comenta que o valor consumido diariamente pode ultrapassar os 15 milhões de dólares. Outras limitações que tangem a esse assunto, como segurança, já foram abordadas nesse capítulo e não é necessária a abordagem nessa seção.

\section{3. Blockchains Economics}

Está seção abordará uma revisão da recente literatura sobre a economia do ecossistema do Bitcoin, apresentando o papel da oferta e da demanda por Bitcoins, sua função como moeda em uma economia, apresentando associações com problemas que os papéis-moedas enfrentam atualmente, como inflação e deflação, além de apresentar a abordagem de alguns autores quanto à forma de determinar quais são as variáveis que impactam o valor de mercado do Bitcoin. 


\subsection{1 \\ Funções da Moeda}

Fernando Cardim (2015) apresenta que uma moeda, como padrão monetário, possui três funções básicas: meio de troca, unidade de conta e reserva de valor. Em torno dessas funções, é bastante discutido se o Bitcoin atende às três funções básicas dadas as moedas, a fim de rotulá-lo certamente como uma forma de moeda.

Ulrich (2016, p. 93) defende o Bitcoin como moeda argumentando que "as três funções da moeda não emergem instantaneamente no momento em que um bem passa a ser utilizado como meio de troca". O autor, nesse caso, busca argumentar que o Bitcoin passa a atender às três funções conforme cresce sua liquidez, popularização e poder de compra ao longo do tempo. Ulrich (2016, p. 95) se estende dizendo que o Bitcoin está em um processo de monetização.

Por outro lado, há autores menos otimistas quanto ao papel de Bitcoin como moeda, como Yermack (2013), que destaca fragilidades da criptomoeda na tentativa de atender às três funções clássicas da moeda. Com relação à função de meio de troca - principal função destacada no paper de Satoshi Nakamoto (2008) Yermack (2013, p. 2) destaca que, apesar do aumento da aceitação do Bitcoin como meio de pagamento, seu mercado e sua aceitação mundial ainda são minúsculos. Yermack (2013, p. 10) ainda afirma que a maioria das transações realizadas em Bitcoins são realizadas por fins especulativos e não para aquisição de bens e mercadorias.

Quanto à função de unidade de conta, Yermack (2013, p. 2) destaca que a volatilidade e a quantidade de decimais do Bitcoin prejudicam sua utilização por compradores e vendedores. Apesar disso, Ulrich (2016, p. 89) observa a divisibilidade do Bitcoin como uma fortaleza e uma arma contra a deflação, visto que a oferta de Bitcoins é fixa no longo prazo.

Em termos de reserva de valor, ou seja, da capacidade de preservar o poder de compra futuro, Yermeck $(2013$, p. 14) destaca a fragilidade das chamadas "carteiras digitais". Dwyer (2014, p. 84) explica que tais carteiras não contêm Bitcoins, mas sim monitoram o saldo de Bitcoins, além de permitir o envio e o recebimento dos mesmos. É nessa carteira que é monitorada as chaves pública e privada do usuário. A questão em torno das fragilidades das carteiras digitais é a questão de segurança, pois, caso seja obtida a chave privada de um usuário, é 
possível fazer a transferência dos Bitcoins sem a possibilidade de recuperação. Outro grande problema que acompanha a função de reserva de valor é a volatilidade da série de tempo do Bitcoin (Yermeck, 2013, p. 14).

Não é unanimidade no meio acadêmico considerar o Bitcoin atualmente como uma moeda, no entanto, o processo para sua monetização pode ser considerado mais lento vis-à-vis as demais moedas fiduciárias, visto que não há imposição governamental pela moeda, dependendo do aumento de sua aceitação e liquidez para que conquiste seu papel como uma moeda.

\subsection{2}

Oferta de Bitcoins

Diferentemente da forma que gerada pelos governos, através de um banco central, a injeção de novos Bitcoins em uma economia é dada pela mineração, como destaca Dwyer (2014, p. 83): "Bitcoins são criados pela solução de problemas computacionais pelos mineradores". Através da mineração, as transações na Blockchain são validadas pelos chamados mineradores, os quais recebem novos Bitcoins e uma taxa pelo serviço prestado como incentivo. No entanto, com o passar do tempo, a dificuldade em criar novos Bitcoins aumenta, visto que a solução para os problemas computacionais se torna cada vez mais difícil. Ulrich (2016, p. 69) apresenta que a oferta de Bitcoins será fixa em 21 milhões e que esse número será alcançado até 2140, mas que cerca de 90\% desse valor já estará disponível em 2022. A inelasticidade da oferta de Bitcoins é tratada como uma vantagem de combate à inflação por alguns autores, como Albuquerque e Callado (2016) e Ulrich (2016). 


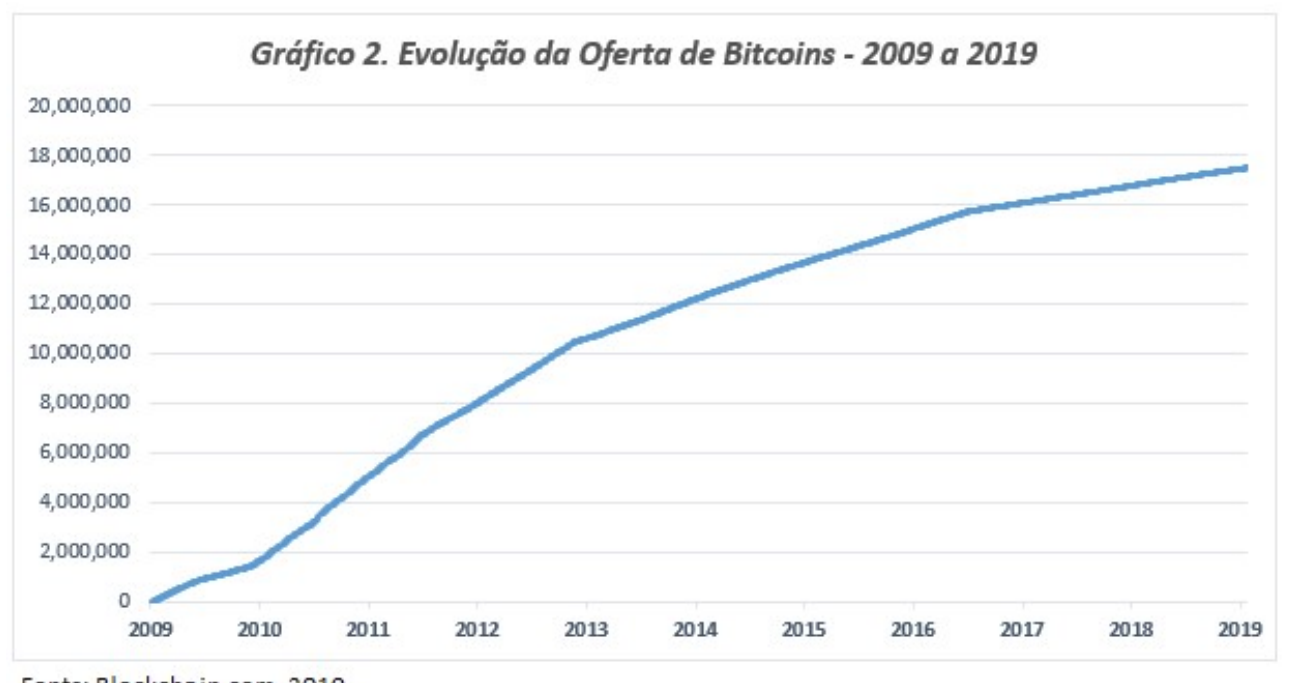

Fonte: Blockchain.com, 2019

\subsection{3 \\ Demanda por Bitcoins}

Diferentemente da curva de oferta muito bem definida, as grandes oscilações pelo lado da demanda têm gerado grande volatilidade (Ulrich, 2016, p. 94). Duas das principais razões para a demanda de uma criptomoeda são destacadas por Dwyer (2014) como o baixo custo de transação e o anonimato. A possibilidade de uma transação sem a necessidade de um intermediário possibilita significantemente a redução de custos. Ao mesmo tempo, o Bitcoin apresenta a privacidade na realização de uma transação que, por muitas vezes, era possível somente com a realização de uma transação com dinheiro físico. Já foram destacadas nesse trabalho as vantagens desses dois pontos, como possibilitar transações que não eram vantajosas anteriormente e possibilitar o combate contra a opressão de minorias.

Além desses pontos, uma grande vantagem comparativa do Bitcoin contra as demais moedas fiduciárias é que é uma forma mais barata de transacionar moedas do que as opções disponíveis para os consumidores atualmente (Dwyer, 2014, p. 90). Seguindo esse raciocínio, é potencialmente mais barato transferir fundos através de Bitcoins do que ficar exposto ao custo de obtenção de moeda estrangeira. Além disso, é possível evitar controles de capitais e sanções governamentais de moeda, como argumentam Ulrich (2016, p. 26) e Dwyer (2014, p. 90). 
No entanto, existem fatores que atualmente diminuem a demanda por Bitcoins, como a criptomoeda não ser vista como reserva de valor. Dwyer (2014, p. 84) destaca que, diferentemente de algumas moedas, onde alguns recursos utilizados na produção de uma moeda podem ser resgatados em alguma quantidade fixa de um ativo com valor, como o ouro, os recursos utilizados na criação de um Bitcoin - em sua maior parte, capacidade computacional e energia - não estão mais disponíveis assim que a criptomoeda é produzida.

\subsection{4}

\section{Inflação, Deflação e Política Monetária}

Albuquerque e Callado (2015, p. 11) argumentam que a prova do esforço (ou proof-of-work) é a única maneira efetiva de criar Bitcoins e que sua oferta é fixada no longo prazo, sendo assim, surtos inflacionários são improváveis. Os autores se estendem afirmando que, ao contrário das moedas fiduciárias, onde o governo pode tomar decisões arbitrárias para criar inflação, os Bitcoin são gerados de acordo com um modelo matemático que permite previsões precisas sobre a quantidade de moeda sobre um período. Não há, nesse caso, um banco central que regule a oferta de Bitcoins em uma economia.

Por outro lado, é muito discutida a questão de deflação no cenário do Bitcoin, onde a oferta de moeda é fixa. Nesse caso, não é interpretada como contração da base monetária, mas sim como aumento do poder de compra de uma unidade. Albuquerque e Callado (2015, p. 11) apresentam esse problema dizendo que uma espiral deflacionária seria inevitável, pois os portadores da moeda as manteriam em posse, observando que a moeda se apreciaria no futuro devido a sua base fixa de moeda ofertada. No entanto, Ulrich (2016, p. 69) argumenta que a perfeita divisibilidade da moeda poderia impedir o surgimento de qualquer efeito deflacionário.

Observado o tratamento de questões relacionadas à política monetária no contexto do Bitcoin, cabe agora apresentar como funcionam tais políticas, visto que não existe um banco central atuando nos bastidores, controlando a oferta disponível da moeda na economia. 
Ulrich (2016, p. 77) destaca que a independência monetária do Bitcoin passa por duas características: a meta de oferta monetária assintótica (MOMA) e pela prova de esforço (ou proof-of-work). A MOMA se refere a uma política monetária próxima de um limite, mas nunca alcançada. A prova de esforço garante a descentralização e a independência da moeda de um banco central. Ulrich (2016, p. 77) argumenta, ainda, que essas duas características geram três fenômenos monetários, são eles:

i) agentes econômicos racionais mantêm encaixes em Bitcoins mesmo não tendo nenhum passivo denominado em Bitcoins; ii) o mercado estabelece as taxas de câmbio e de juros, sem exceção; e iii) é altamente improvável o aparecimento de reservas fracionadas.

Segundo essa ótica, o Bitcoin teria uma política monetária independente e liberdade total dos fluxos de capitais, de modo que os agentes podem decidir livremente o saldo de Bitcoins que querem manter, impulsionados pelas vantagens futuras que essa moeda possa gerar, podendo levar à sua valorização.

\subsection{5 Determinantes do Preço do Bitcoin}

Esta seção retoma o cerne da pesquisa proposta nesse trabalho, buscando apontar quais seriam as variáveis que nos ajudariam a entender as flutuações de preços do Bitcoin.

Alguns autores como Buchholtz et al. (2012), Bouoiyour e Selmi (2015) e Ciaian, Rajcaniova e Kancs (2016) fundamentaram seus modelos com as forças de mercado, oferta e demanda por Bitcoin, para entender o preço da criptomoeda. Em suas hipóteses, utilizando a clássica função de Teoria Quantitativa da Moeda, era esperado que o nível geral de preços de bens e serviços e o tamanho da economia do Bitcoin teriam efeitos positivos sobre o preço do Bitcoin, e a velocidade da moeda e o número de Bitcoins ofertados teriam impacto negativo sobre os preços. Segundo Ciaian, Rajcaniova e Kancs (2016), o número de Bitcoins ofertados, por ser predefinido, é uma variável semi-exógena, assim, o efeito do seu coeficiente deveria ser pequeno ou estatisticamente não significante. 
Kristoufek (2013), Ciaian, Rajcaniova e Kancs (2016) e Lin e Wang (2016) apresentam modelos onde a atratividade dos investidores e o reconhecimento público começam a figurar como variáveis importantes na determinação do preço da criptomoeda. Utilizando ferramentas de buscas e redes sociais como proxy, como Google, Wikipedia e Twitter, onde esperava-se que o reconhecimento público teria efeito positivo sobre os preços e que poderiam tomar a forma de um efeito negativo caso as notícias sobre o Bitcoin fossem negativas.

Van Wijk (2013), Ciaian, Rajcaniova e Kancs (2016) e Lin e Wang (2016) apresentam modelos os quais incluem variáveis macroeconômicas globais a fim de medir se existia alguma relação com a série de preços do Bitcoin, utilizando taxa de juros e índices de ações. Nesse caso, busca-se puramente a existência de uma ligação entre indicadores macroeconômicos mundiais com a flutuação do preço da criptomoeda.

Por fim, Lin e Wang (2016) apresentam uma abordagem mais recente, incluindo variáveis relacionadas à tecnologia do Bitcoin, como a dificuldade de minerar. Os autores esperavam que tais fatores tivessem um impacto positivo sobre o preço do Bitcoin e, como é esperado que os custos para minerar tendam a diminuir devido ao aumento de eficiência ao longo dos anos, espera-se que esse impacto diminua ao longo do tempo.

O presente trabalho elabora seu modelo a partir de todos os modelos anteriores, buscando atestar o efeito e comparar resultados a partir do estudo da série de preços de seu período mais volátil até o presente o momento (2017 a 2018), o qual não é retratado por nenhum dos autores que escreveram anteriormente sobre o tema. 


\section{3 Metodologia}

Este capítulo busca apresentar a especificação do modelo proposto para entender os determinantes do preço de mercado do Bitcoin, além de detalhar a forma que os dados foram obtidos e o software utilizado para cálculo do modelo. Outro grande objetivo é descrever a metodologia econométrica utilizada para analisar os dados que, posteriormente, serão utilizados como fundamento para interpretação dos resultados obtidos através desse modelo.

\section{1 Especificação do Modelo}

O modelo proposto foi fundamentado nos estudos previamente realizados por Buchholtz et al. (2012), Kristoufek (2013), Van Wijk (2013), Bouoiyour e Selmi (2015), Ciaian, Rajcaniova e Kancs (2016) e Lin e Wang (2016), onde cada um dos autores acrescentou uma gama de variáveis buscando entender suas interações com a série de preço do Bitcoin. Dessa forma, unificamos cada um dos componentes fundamentados pelos autores em um único construto, a fim de entender se as diversas abordagens apresentadas conseguem explicar os movimentos de preços da criptomoeda nos dias atuais.

A especificação do modelo pode ser observada pela equação 1 , na qual se propõe que o preço do Bitcoin é impactado por 4 grupos de variáveis: forças de mercado (FM), reconhecimento público $(\mathrm{R})$, macroeconomia global (MG) e tecnologia (T).

$$
P B_{t}=\beta_{1}+\beta_{2} F M_{t}+\beta_{3} R_{t}+\beta_{4} M G_{t}+\beta_{5} T_{t}+\varepsilon_{t}
$$

A variável $\varepsilon$ representa o termo de erro estocástico da série e o subscrito $\mathrm{t}$ representa o tempo. 


\section{2 \\ Variáveis e Dados}

O presente estudo econométrico se propôs a analisar o período de maior volatilidade da série de preços do Bitcoin, o qual delimita-se entre janeiro de 2017 e dezembro de 2018. É importante ressaltar que a literatura sobre análises econométricas sobre esse período ainda é escassa. A Tabela 2 apresenta a média, a variância e o desvio-padrão do período analisado e da parcela da série que não está no escopo do estudo. É possível visualizar a diferença abrupta dos momentos da série entre os dois períodos apresentados.

\begin{tabular}{|c|c|c|c|}
\hline \multicolumn{1}{|c}{ Ano } & Média & Variância & Desvio-Padrão \\
\hline $2009-2016$ & 196.09 & $62,727.38$ & 250.45 \\
\hline $2017-2018$ & $5,784.88$ & $14,411,111.18$ & $3,796.20$ \\
\hline
\end{tabular}

Tabela 2. Média, Variância e Desvio-Padrão da Série do Bitcoin em Dólares Americanos Fonte: Blockchain.com, 2019

O estudo utilizou a série da taxa de conversão entre Bitcoins e dólares americanos, variável dependente do modelo, onde a fonte de dados secundária foi extraída do site Blockchain.com. Tal fonte de dados já foi utilizada por outros autores, como em Buchholtz et al. (2012) e Lin e Wang (2016).

Com relação ao grupo de variáveis de forças de mercado do Bitcoin expressado no modelo, foram selecionadas quatro variáveis que têm impacto, direto ou indireto, sobre a oferta e demanda de Bitcoins. São elas: a oferta de Bitcoins, receita de mineração, custo por transação e número total de transações diárias.

A oferta de Bitcoins representa a quantidade de Bitcoins que já foram mineradas, em outras palavras, é o montante total da criptomoeda que está disponível na economia global em um determinado momento. A receita de mineração representa o valor total de taxas de transação e recompensas remuneradas ao mineradores pela prova de esforço realizada na criação de novos blocos da cadeia do Bitcoin. O custo por transação na rede é denominado pela divisão da receita dos mineradores pelo número de transações. O número total de transações diárias representa simplesmente o número de transações confirmadas na 
rede diariamente. Os dados das quatro variáveis foram extraídos do site Blockchain.com, da mesma forma que a série de preços do Bitcoin.

O termo reconhecimento público foi medido por uma proxy relacionada às buscas da palavra Bitcoin na ferramenta de pesquisas Google. Essa proxy também foi utilizada por Kristoufek (2013) e Lin e Wang (2016). Lin e Wang (2016, p. 54) argumentavam que a intensidade de buscas se concentra no reconhecimento impulsionado pela recuperação de informações, indicando a intenção de aprender sobre o Bitcoin. Os dados foram retirados do Google Trends, através da busca do termo de pesquisa "Bitcoin", dos anos de 2017 e 2018 no mundo. Os dados extraídos não representam o volume absoluto de buscas, mas sim são apresentados em uma escala de 0 a 100, onde é refletida a quantidade de buscas realizadas de um termo particular em relação ao número total de buscas realizadas no Google.

Quanto ao grupo de variáveis que representam a macroeconomia global, foram utilizadas 4 variáveis para tentar capturar se o dinamismo das economias globais têm impacto no comportamento da série de preço do Bitcoin. Dentre as variáveis, dois índices de mercados foram utilizados: Dow Jones e Nikkei 225. O índice Dow Jones é composto por uma média ponderada dos preços de 30 ações significativas da New York Exchange (NYSE) e da National Association of Securities Dealers Automated Quotations (NASDAQ), mensurados em dólares americanos. Van Wijk (2013, p. 5) destaca que esse índice pode ser utilizado como indicador da economia dos Estados Unidos. O índice Nikkei 225 é composto pela média ponderada de 225 grandes companhias do Japão listadas na Tokyo Stock Exchange, mensuradas em yen (moeda japonesa). Van Wijk (2013, p. 5) destaca esse índice como indicador da economia do Japão e de diversos países da Ásia Oriental. Os dados foram coletados através da plataforma digital da Investing.com.

As demais variáveis que compõem esse grupo se referem a uma commodity, o índice futuro dos preços do petróleo bruto na West Commodity Index (WTI), mensurado em dólares americanos, e a taxa de juros diária efetiva dos fundos federais dos Estados Unidos. A intenção da inclusão dessas variáveis no modelo é saber se a dinâmica da maior matriz energética do mundo e das ferramentas de política monetária da maior economia mundial tem impacto sobre os preços da criptomoeda. Os dados do índice futuro do preço do petróleo bruto na WTI foi extraído do site Investing.com e a série da taxa de juros americana foi obtida através do site do banco central americano (Federal Reserve). 
O termo tecnológico do modelo foi inspirado no trabalho de Lin e Wang (2016) que acreditavam que fatores tecnológicos influenciavam na tomada de preço da criptomoeda. Uma proxy utilizada é a dificuldade de mineração que consiste na medida relativa da dificuldade de encontrar um novo bloco. Lin e Wang (2016, p. 52) se estendem argumentando que essa variável também é capaz de captar a robustez do sistema. Os dados foram extraídos através do site Blockchain.com.

Todos os dados extraídos foram compilados no software "R Project for Statistical Computing", o qual foi utilizado para aplicação do ferramental econométrico que será abordado na seção seguinte.

\section{3}

\section{Abordagem Econométrica}

A partir dessa seção serão apresentadas as ferramentas de análise utilizadas para entender a relação das variáveis independentes, propostas na especificação do modelo, com a série temporal do Bitcoin. Buscou-se utilizar dois modelos bastante disseminados no estudo de séries de tempo e em pesquisas na área de criptomoedas, além dos respectivos testes necessários à sua implementação. Os modelos são: Vetor de Correção de Erros (VECM) e procedimento Autoregressivo de Defasagens Distribuídas (ARDL). O Modelo do Vetor Autoregressivo (VAR) foi considerado a priori, mas durante a análise dos testes necessários à sua especificação, as variáveis não atenderam a hipótese de estacionaridade exigida pelo modelo.

\subsection{1}

\section{Testes de Raiz Unitária}

Em análises de séries temporais, uma das primeiras e principais avaliações a serem realizadas é quanto a sua estacionariedade. Em linhas gerais, as séries de tempo estacionárias possuem primeiro e segundo momentos que não variam ao longo do tempo ${ }^{4}$, ou seja, sua esperança e sua covariância são independentes do

${ }^{4}$ ZIVOT, E.; WANG, J. Modeling financial time series with S-Plus R ०. [S.1.]: Springer Science \&Business Media, 2005. v. 191, p. 53. 
tempo. Esse conceito é importante para a realização de inferências sobre os parâmetros estimados e para a adequação de tais séries em modelos válidos.

Uma alternativa para avaliar a estacionaridade da série de tempo é utilizar testes de raiz unitária, os quais podem determinar se os dados de tendência devem ser tratados em primeira diferença ${ }^{5}$, também expressos pelo termo $I(1)$, ou regredidos em funções determinísticas do tempo para tornar os dados estacionários (Zivot, 2005, p. 111). Se as séries forem $I(1)$, técnicas de cointegração podem ser utilizadas para modelar relações de longo prazo. Tais técnicas se apresentaram necessárias ao longo das análises de dados e serão apresentadas na seção seguinte.

O primeiro teste aplicado consiste no teste Dickey-Fuller Aumentado (ADF). Usualmente, a hipótese nula do teste diz que a série não tem raiz unitária, o que indica que a série é estacionária, e a hipótese alternativa apresenta que a série possui raiz unitária. A formulação da regressão do teste ADF para essas hipóteses é dada pela equação 2 .

$$
\Delta y_{t}=\beta^{\prime} D_{t}+\pi y_{t-1}+\sum_{j=1}^{p} \varphi_{j} \Delta y_{t-j}+\varepsilon_{t}(2)
$$

Nela, $D_{t}$ é um vetor dos termos determinísticos (constante, tendência etc.). O $p$ é o termo que representa o número de defasagens, $\Delta y_{t-j}$ é usado para aproximar os erros da estrutura ARMA. O valor $p$ é definido para que o erro $\varepsilon_{t}$ seja serialmente não correlacionado. $\mathrm{O}$ termo de erro é assumido como homocedástico. Os termos $\beta^{\prime}, \pi, e \varphi_{j}$ são os coeficientes relacionados às variáveis. Quando o valor de $\pi=0$, isso implica na não rejeição da hipótese nula de não existência de raiz unitária, ou seja, que a série é estacionária. Essa relação é testada sobre a estatística definida na equação 3 .

$$
A D F_{n}=\frac{T \hat{\pi}}{\left(1-\widehat{\varphi_{1}}-\cdots-\widehat{\varphi_{p}}\right)}
$$

\footnotetext{
5 A diferenciação de uma equação é uma expressão da variável dependente $\left(y_{t}\right)$ a seus valores passados. A diferença em primeira ordem, ou $I(1)$, é denominada quando a primeira defasagem da variável dependente aparece na equação $\left(y_{t-1}\right)$ (HAMILTON, 1994, p. 1).
} 
O número de defasagens utilizadas para cada variável dependente nesse teste foi determinado pelo Critério de Informação de Akaike (AIC), que pode ser observado na equação 4 , onde $\ln \widehat{\sigma^{2}}$ é o logarítimo niperiano da variância dos resíduos, $T$ é o número de observações e $n$ é a soma da ordem do modelo autoregressivo (AR) e do modelo de médias móveis (MA).

$$
A I C=\ln \widehat{\sigma^{2}}+n \frac{2}{T}
$$

Outro teste que é habitualmente utilizado em conjunto com o teste DickeyFuller Aumentado, e também é utilizado para testar estacionariedade da série, consiste no KSPP. Diferentemente do teste anterior, que verifica a existência de raiz unitária para definir estacionariedade, o KPSS é um teste que verifica diretamente se a série é estacionária ou não, sob a hipótese nula de estacionariedade e hipótese alternativa de a série ser não-estacionária. A estatística do teste é definida pelo multiplicador de Lagrange (LM) e pode ser observada pela equação 5, onde $T$ é o número de observações, $S_{t}^{2}$ são os resíduos da regressão e $\gamma^{2}$ é uma estimativa consistente da variância de longo prazo dos resíduos.

$$
K P S S=\frac{\left(T^{-2} \sum_{t=1}^{T} S_{t}^{2}\right)}{\gamma^{2}}
$$

A partir da aplicação desses testes poderemos escolher o modelo mais adequado para a aplicação do modelo especificado na seção 3.1. Se todas as séries encontradas forem estacionárias, poderíamos utilizar o Vetor Autoregressivo (VAR) como modelo para analisar os dados. No entanto, analisando o resultado dos testes, que serão apresentados no próximo capítulo, esse modelo não seria adequado para interpretar o modelo proposto.

\subsection{2}

\section{Teste de Cointegração}

Quando se observa que as variáveis não são estacionárias em nível ( $I(0)$ ), mas são estacionárias em suas primeiras diferenças ( $I(1)$ ), apresenta-se a 
necessidade de analisar se existe cointegração entre as variáveis. Em outras palavras, uma série não-estacionária possui cointegração se existe alguma combinação linear, a qual é estacionária. Essa relação pode ser representada pela equação $6, \beta^{\prime}$ onde é o vetor de cointegração.

$$
\beta^{\prime} Y_{t}=\beta_{1} y_{1 t}+\cdots+\beta_{n} y_{n t} \sim I(0)(6)
$$

Se alguns elementos do vetor de cointegração forem iguais a zero, somente o subconjunto da série de tempo que não for zero é cointegrado. A combinação linear $\beta^{\prime} Y_{t}$ é geralmente motivada pela teoria econômica e referida como relação de equilíbrio de longo prazo (Zivot, 2005, p. 433).

No presente estudo, as variáveis foram normalizadas seguindo a orientação de Zivot (2005, p. 433) que argumentava que alguma hipótese de normalização é necessária para identificar o vetor de cointegração.

O teste de cointegração cobre duas situações: i) Existe pelo menos um vetor de cointegração, e ii) Existe a possibilidade de $0 \leq \mathrm{r}<\mathrm{n}$ vetores de cointegração.

O teste aplicado nesse estudo é o de Johansen (1988), o qual é utilizado para identificar o rank da matriz $\Pi$, determinando o número de vetores de cointegração, no modelo Vetor de Correção de Erros (VECM) apresentado na seção seguinte. A vantagem dessa metodologia consiste na possibilidade do VECM ser estimado simultaneamente aos vetores de cointegração.

Johansen (1988) apresenta dois testes para identificar cointegração. As estatísticas de ambos os testes são baseadas na estimação por máxima verossimilhança.

O primeiro teste é denominado como traço e suas hipóteses e estatísticas são apresentadas na equação 7.

$$
\begin{aligned}
H_{0}(r): r & =r_{0} \text { vs. } H_{1}\left(r_{0}\right): r>r_{0} \\
L R_{\text {traço }}\left(r_{0}\right) & =-T \sum_{i=r_{0}+1}^{n} \ln \left(1-\hat{\gamma}_{i}\right)
\end{aligned}
$$

$\mathrm{Na}$ estatística acima, $\hat{\gamma}_{i}$ representa os autovalores da matriz $\Pi$. Se o rank da matriz $\Pi$ é igual a $r_{0}$, os autovalores devem estar próximos de zero, assim, a 
estatística do teste deve ser pequena. Mas, caso o rank da matriz П seja maior que $r_{0}$, alguns autovalores serão diferentes de zero e a estatística terá alto valor.

O segundo teste consiste em determinar o número de vetores de cointegração e é denominado como teste do máximo autovalor. Ele é realizado de forma sequencial, por exemplo, se o primeiro teste $H_{0}\left(r_{0}\right)=0$ contra $H_{1}\left(r_{0}>0\right)$, se não rejeitarmos a hipótese nula, conclui-se que não existe vetor de cointegração entre as $n$ variáveis da variável dependente $Y_{t}$; caso contrário, seria concluído que existe pelo menos um vetor de cointegração. Esse teste é realizado até a hipótese nula não ser rejeitada. As hipóteses e a estatística de teste podem ser observadas na equação 8.

$$
\begin{gathered}
H_{0}\left(r_{0}\right): r=r_{0} \text { vs. } H_{1}\left(r_{0}\right): r_{0}=r_{0}+1 \\
L R_{\max }\left(r_{0}\right)=-T \ln \left(1-\hat{\gamma}_{r_{0}+1}\right)(8)
\end{gathered}
$$

Apesar da complexidade, os dois testes apresentados por Johansen (1988) são amplamente utilizados na literatura para identificar cointegração. De forma sintética, o teste do traço verifica a existência de cointegração, enquanto o teste de máximo autovalor identifica a quantidade de cointegrações existentes.

Para determinar se é necessária a inclusão de uma constante ou de um termo determinístico, seguimos o princípio de Pantula (1989), que consiste em testar sequencialmente as hipóteses do modelo menos restritivo (sem termo determinístico) até o modelo mais restritivo (incluindo o termo determinístico). $\mathrm{O}$ processo ocorre até o modelo preferido ser identificado pela primeira vez que a hipótese nula é rejeitada.

\subsection{3}

\section{Modelo Vetor de Correção de Erros (VECM)}

O primeiro modelo utilizado consiste na estimação através do Modelo Vetor de Correção de Erros (VECM). Esse modelo é considerado uma generalização do Vetor Autoregressivo (VAR), o qual incorpora correções de longo prazo de modo 
que tanto as dinâmicas de curto prazo quanto de longo prazo possam ser estudadas. Esse vetor é especificado na equação 9.

$$
\Delta Y_{t}=\Phi^{\prime} D_{t}+\Pi Y_{t-1}+\Gamma_{1} \Delta Y_{t-1}+\cdots+\Gamma_{p-1} \Delta Y_{t-p+1}+\varepsilon_{t}(9)
$$

Nesta equação, $D_{t}$ consiste no termo determinístico, П é a matriz de impacto de longo prazo e $\Gamma_{k}$ são as matrizes de impacto no curto prazo. Para a utilização desse modelo, as variáveis não devem ser estacionárias em níveis e deve existir ao menos um vetor de cointegração.

Com todas as premissas atendidas, podemos observar o rank da matriz $\Pi \mathrm{e}$ verificar o número de cointegrações a partir da realização dos testes de cointegração de Johansen e, assim, estimar os resultados do Modelo Vetor de Correção de Erros (VECM) a partir da máxima verossimilhança.

\subsection{4 Procedimento Autoregressivo de Defasagens Distribuídas (ARDL)}

A estimação pelo Procedimento Autoregressivo de Vantagens Distribuídas (ARDL) foi o segundo modelo escolhido para análise dos resultados dos determinantes do preço do Bitcoin. Esse modelo possui uma grande vantagem sobre o VAR e o VECM, visto que é possível ter estimadores consistentes de longo prazo que são assintoticamente normais, independente se as variáveis são estacionárias em nível $I(0)$ ou se são estacionárias em primeira diferença $I(1)$. Para essa abordagem, seguimos Pesaram e Shin (1999), os quais especificaram o modelo geral representado nas equações 10 e 11 .

$$
\begin{gathered}
y_{t}=\alpha_{0}+\alpha_{1} t+\sum_{i=0}^{p} \phi_{i} y_{t-i}+\beta^{\prime} x_{t}+\sum_{i=0}^{q-1} \beta_{i}^{*} \Delta x_{t-1}+u_{t}(10) \\
\Delta x_{t}=P_{1} \Delta x_{t-1}+P_{2} \Delta x_{t-2}+\cdots+P_{s} \Delta x_{t-s}+\varepsilon_{t}(11)
\end{gathered}
$$

Aqui, $x_{t}$ é o vetor das variáveis que não são cointegráveis entre elas, $u_{t}$ e $\varepsilon_{t}$ são os distúrbios serialmente não correlacionados com média zero e covariância constante, e $P_{i}$ é a matriz de coeficientes no qual o processo do vetor autoregressivo 
em $\Delta x_{t}$ é estável. Para a escolha do número de defasagens, mativemos o resultado do critério de informação de Akaike.

Em seguida, é aplicado o teste de bandas que testa a existência de relação entre as variáveis em nível, independente se as variáveis são estacionárias em nível, estacionária em diferenças ou ambos os casos. As bandas desse teste são definidas assumindo que os regressores em um limite da banda são puramente estacionários em primeira diferença e, no segundo limite, os regressores são puramente estacionários em nível. Definidas as bandas do teste, são identificados os valores críticos para aplicação desse teste.

A partir da aplicação da metodologia apontada nesse capítulo, serão apresentados os resultados das estimações dos modelos e dos testes, assim como a interpretação do resultados vis-à-vis aos resultados de outros autores. 


\section{4 Resultados}

O presente capítulo tem por objetivo expor os resultados dos modelos propostos, além de apresentar sua interpretação à luz do referencial teórico e da metodologia introduzida nos capítulos anteriores, comparando com resultados encontrados por outros autores.

\section{1.}

\section{Análise de Estacionariedade}

São propostos nesse estudo dois testes para analisar a estacionariedade das séries de dados, em nível e em suas primeiras diferenças, que serão utilizadas para estimação do modelo conceitual das variáveis que podem influenciar a série de preços do Bitcoin.

O primeiro modelo é o teste Dickey-Fuller Aumentado (ADF), o qual testa em sua hipótese a presença de raiz unitária na série. O resultado desse teste auxilia em determinar se os dados devem ser tratados em níveis ou em diferenças. A hipótese nula desse teste explicita a ausência de raiz unitária, o que indica que a série é estacionária. Na Tabela 3 é apresentado o teste para as variáveis do arcabouço teórico proposto no estudo, onde todas as variáveis são testadas com a ausência de um termo determinístico, com a presença de constante e com a presença de um termo de tendência. $\mathrm{O}$ número de defasagens utilizadas no teste foi determinado pelo Critério de Informação de Akaike, o qual resultou em 6 defasagens para o presente modelo. 


\begin{tabular}{lccc}
\hline \hline \multicolumn{1}{c}{ Variáveis } & Sem Constante e Tendência & Com Constante & Com Tendência \\
\hline Preço_Bitcoin & -0.548 & -1.493 & -0.955 \\
Buscas_Google & $-2.163 * *$ & $-4.979 * * *$ & $-5.481^{* * *}$ \\
Dificuldade_Bitcoin & 1.368 & -0.61 & -0.944 \\
Oferta_Bitcoin & 22.928 & -0.728 & -2.216 \\
Receita_Bitcoin & -0.916 & -1.779 & -1.59 \\
Custo_Bitcoin & -0.883 & -1.844 & -1.548 \\
Transação_Bitcoin & -0.738 & $-3.995 * * *$ & $-4.247 * * *$ \\
Juros_EUA & 2.553 & -0.019 & $-3.374 * *$ \\
Dow_Jones & 0.526 & -1.835 & -1.199 \\
Nikkei_225 & -0.014 & -1.682 & -1.317 \\
Petroleo_WTI & -0.519 & -0.788 & 0.285 \\
Obs: ${ }^{*} p<0.10, * * p<0.05 . e{ }^{* * *} p<0.01$ & &
\end{tabular}

Tabela 3. Teste Dickey-Fuller Aumentado (ADF) nas séries em nível.

Entre as variáveis do modelo, é possível observar que somente três séries de dados possuem raiz unitária em pelo menos uma de suas modelagens, ou seja, indicam que as séries são estacionárias em nível. São elas: as pesquisas no Google, o número de transações de Bitcoins e a taxa de juros diária americana. Isso indica que a estimação da regressão pelo vetor autoregressivo (VAR) não seria apropriada caso seja proposto estimar todas as variáveis do modelo especificado. Como o modelo reduzido somente apresentaria uma regressão com as três variáveis estacionárias em nível, não seria atendida integralmente a proposição desse estudo, que é entender a interação dos 4 grupos de variáveis apresentados na seção 3.1. Por esse motivo, decidiu-se utilizar outras abordagens para encontrar os coeficientes da regressão.

Em seguida, é aplicado o teste ADF novamente utilizando as séries de dados das variáveis em suas primeiras diferenças. A Tabela 4 sintetiza o resultado dos testes, seguindo o mesmo número de defasagens do teste ADF e apresentando os resultados para modelos com constante e tendência. 


\begin{tabular}{lccc}
\hline \hline \multicolumn{1}{c}{ Variáveis } & Sem Constante e Tendência & Com Constante & Com Tendência \\
\hline Preço_Bitcoin & $-12.84^{* * *}$ & $-12.822^{* * *}$ & $-12.85^{* * *}$ \\
Buscas_Google & $-18.631^{* * *}$ & $-18.581^{* * *}$ & $-18.850^{* * *}$ \\
Dificuldade_Bitcoin & $-12.873^{* * *}$ & $-13.061^{* * *}$ & $-13.053^{* * *}$ \\
Oferta_Bitcoin & -0.707 & $-9.271^{* * *}$ & $-9.635^{* * *}$ \\
Receita_Bitcoin & $-15.590^{* * *}$ & $-15.57^{* * *}$ & $-15.592^{* * *}$ \\
Custo_Bitcoin & $-17.502^{* * *}$ & $-17.504^{* * *}$ & $-17.532^{* * *}$ \\
Transação_Bitcoin & $-18.082^{* * *}$ & $-18.145^{* * *}$ & $-18.286^{* * *}$ \\
Juros_EUA & $-14.385^{* * *}$ & $-14.532^{* * *}$ & $-14.551^{* * *}$ \\
Dow_Jones & $-16.251^{* * *}$ & $-16.258^{* * *}$ & $-16.355^{* * *}$ \\
Nikkei_225 & $-14.831^{* * *}$ & $-14.816^{* * *}$ & $-14.858^{* * *}$ \\
Petroleo_WTI & $-14.852^{* * *}$ & $-14.844^{* * *}$ & $-14.912^{* * *}$ \\
Obs: ${ }^{*} p<0.10, * * p<0.05 . e^{* * *} p<0.01$ & & \\
\hline
\end{tabular}

Tabela 4. Teste Dickey-Fuller Aumentado (ADF) nas séries em primeira diferença.

Para o resultado do teste ADF nas séries em primeira diferença, podemos observar a presença de raiz unitária em quase todos os casos, somente excluindo a série da oferta de Bitcoins sem constante e sem tendência. Esses resultados nos ajudam a utilizar um modelo alternativo ao VAR, conhecido como vetor de correção de erros (VECM), o qual permite a utilização das séries estacionárias em suas primeiras diferenças.

O segundo teste proposto é o KPSS que, diferentemente do ADF, que testa a presença de raiz unitária, tem como hipótese de teste a estacionariedade da série. Com sua estatística definida pelo multiplicador de Lagrange, podemos observar o resultado dos testes em séries em níveis na Tabela 5. 


\begin{tabular}{lcc}
\hline \hline \multicolumn{1}{c}{ Variáveis } & Com Constante & Com Tendência \\
\hline Preço_Bitcoin & 3.248 & 1.317 \\
Buscas_Google & 1.349 & 0.348 \\
Dificuldade_Bitcoin & 7.532 & 1.374 \\
Oferta_Bitcoin & 8.082 & 0.726 \\
Receita_Bitcoin & 2.108 & 1.123 \\
Custo_Bitcoin & 3.283 & 1.495 \\
Transação_Bitcoin & 2.06 & 0.394 \\
Juros_EUA & 7.785 & 0.635 \\
Dow_Jones & 6.882 & 0.977 \\
Nikkei_225 & 5.433 & 0.847 \\
Petroleo_WTI & 4.964 & 0.736 \\
Obs: Valores críticos a 10\%, 5\% e 1\% são respectativamente 0.463, 0.574 e \\
O.739 para a série com constante e 0.146, 0.176 e 0.216 para a série com \\
tendência.
\end{tabular}

Tabela 5. Teste KPSS nas séries em nível.

Diferentemente dos resultados do teste ADF, os resultados dos testes nas séries em nível apresentam a rejeição à hipótese de estacionariedade, reforçando que a aplicação do VAR, modelo utilizado para estimar séries estacionárias em níveis, não é adequada nesse estudo. A Tabela 6 apresenta os resultados dos testes KPSS para as séries em suas primeiras diferenças.

\begin{tabular}{lcc}
\hline \hline \multicolumn{1}{c}{ Variáveis } & Com Constante & Com Tendência \\
\hline Preço_Bitcoin & 0.135 & 0.052 \\
Buscas_Google & 0.052 & 0.019 \\
Dificuldade_Bitcoin & 0.293 & 0.304 \\
Oferta_Bitcoin & 0.857 & 0.337 \\
Receita_Bitcoin & 0.121 & 0.035 \\
Custo_Bitcoin & 0.132 & 0.049 \\
Transação_Bitcoin & 0.369 & 0.125 \\
Juros_EUA & 0.094 & 0.029 \\
Dow_Jones & 0.266 & 0.072 \\
Nikkei_225 & 0.176 & 0.075 \\
Petroleo_WTI & 0.361 & 0.231
\end{tabular}

Obs: Valores críticos a 10\%, 5\% e 1\% são respectativamente 0.463 , 0.574 e 0.739 para a série com constante e 0.146, 0.176 e 0.216 para a série com tendência.

Tabela 6. Teste KPSS nas séries em primeira diferença. 
É possível observar que somente quatro testes rejeitam a estacionariedade em primeira diferença. São eles: a oferta de Bitcoins com constante, a oferta de Bitcoins com tendência, a dificuldade de mineração de Bitcoins com tendência e os preços do petróleo com tendência.

Em síntese, podemos observar com a aplicação dos dois testes, ADF e KPSS, que o modelo VAR não é o mais adequado a ser aplicado nesse estudo, visto que a estacionariedade em nível das séries de dados não é unânime dentre os resultados dos testes. Por outro lado, a estacionariedade em primeira diferença é vista em todas as variáveis quando observamos os resultados dos testes e suas demais variações dos modelos, incluindo constante e tendência.

Os achados desses estudos corroboram com os resultados recentes encontrados em Ciaian, Rajcaniova e Kancs (2016) e Lin e Wang (2016), que buscaram modelos alternativos observando que as séries utilizadas não apresentavam estacionariedade em nível.

\section{2. Análise de Cointegração}

Para análise de séries não estacionárias em níveis, é necessária a aplicação de um teste de cointegração, a fim de verificar a existência de alguma combinação linear a qual torna a série estacionária.

O teste adotado é conhecido como teste de cointegração de Johansen, o qual, além de testar a presença de cointegração, auxilia na identificação do rank da matriz que será utilizado para estimação dos coeficientes do vetor de correção de erros (VECM).

A detecção de cointegração pelo modelo de Johanssen (1988) apresenta dois testes: o teste do traço e o teste do máximo autovalor. O primeiro teste consiste em detectar a existência de cointegração entre as séries. $O$ segundo consiste em verificar a quantidade de relações de cointegração existentes.

A fim de determinar a necessidade da inclusão de uma constante ou um termo determinístico, é utilizado o princípio de Pantula (1989), que consiste em testar sequencialmente as hipóteses do modelo menos restritivo (sem termo 
determinístico) até o modelo mais restritivo (incluindo o termo determinístico). A Tabela 7 apresenta os resultados dos testes de traço e de máximo autovalor para todos os modelos.

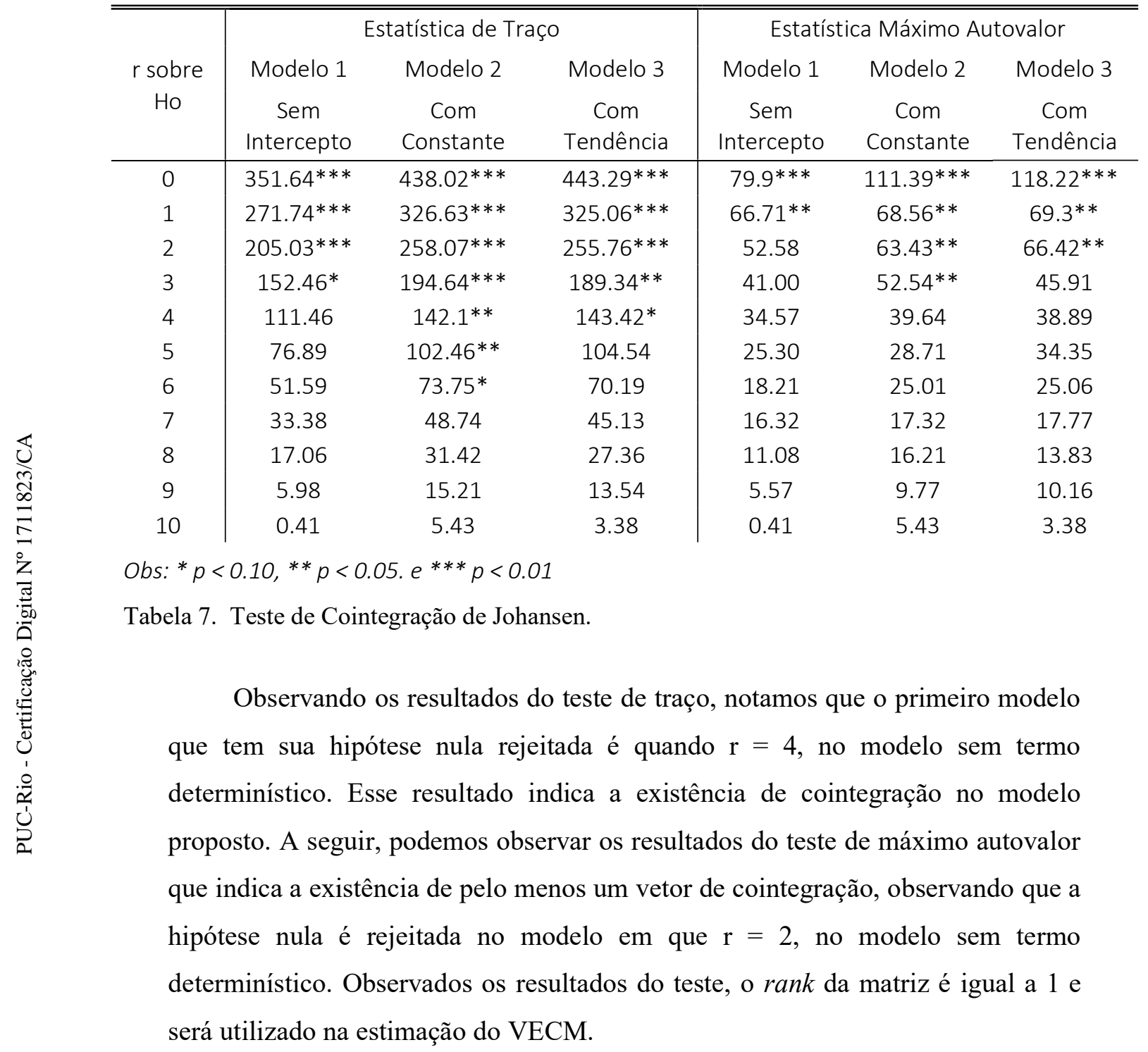

\section{3.}

\section{Análise do Modelo de Correção de Erros}

Ao se observar que as séries de dados possuem cointegração e são estacionárias em primeira diferença, é aplicado o vetor de correção de erros, uma generalização do vetor autoregressivo, o qual incorpora em seu modelo correções 
de longo prazo de modo que tanto as dinâmicas de curto prazo quanto de longo prazo possam ser estudadas.

Utilizando o rank encontrado no teste de cointegração de Johanssen e o princípio de Pantula (1989) para definição do modelo a ser estudado, é possível observar o resultado da estimação na Tabela 8 . O modelo 3 (com tendência) foi escolhido e o rank da matriz é igual a 1.

\begin{tabular}{|c|c|c|c|c|}
\hline & Coeficiente & Desvio Padrão & t valor & $\operatorname{Pr}(>|t|)$ \\
\hline Intercepto $(\alpha)$ & 0.008 & 0.035 & 0.220 & 0.826 \\
\hline Constante & 0.320 & 1.310 & 0.244 & 0.807 \\
\hline$\Delta$ Preço_Bitcoin $_{t-1}$ & -0.077 & 0.056 & -1.381 & 0.168 \\
\hline$\Delta$ Preço_Bitcoin ${ }_{t-2}$ & 0.074 & 0.061 & 1.215 & 0.225 \\
\hline$\Delta$ Preço_Bitcoin ${ }_{t-3}$ & 0.003 & 0.061 & 0.049 & 0.961 \\
\hline$\Delta$ Preço_Bitcoin ${ }_{t-4}$ & 0.186 & 0.061 & 3.076 & $0.002236 * *$ \\
\hline$\Delta$ Preço_Bitcoin ${ }_{t-5}$ & -0.110 & 0.060 & -1.826 & 0.068636 \\
\hline$\Delta$ Buscas_Google $_{t-1}$ & 0.004 & 0.012 & 0.342 & 0.733 \\
\hline$\Delta$ Buscas_Google $_{t-2}$ & -0.003 & 0.012 & -0.257 & 0.797 \\
\hline$\Delta$ Buscas_Google $_{t-3}$ & -0.013 & 0.012 & -1.097 & 0.273 \\
\hline$\Delta$ Buscas_Google $_{t-4}$ & -0.010 & 0.012 & -0.827 & 0.409 \\
\hline$\Delta$ Buscas_Google ${ }_{t-5}$ & -0.005 & 0.012 & -0.451 & 0.652 \\
\hline$\Delta$ Dificuldade_Bitcoin ${ }_{t-1}$ & 0.027 & 0.158 & 0.172 & 0.863 \\
\hline$\Delta$ Dificuldade_Bitcoin $n_{t-2}$ & 0.070 & 0.161 & 0.436 & 0.663 \\
\hline$\Delta$ Dificuldade_Bitcoin ${ }_{t-3}$ & -0.010 & 0.161 & -0.061 & 0.951 \\
\hline$\Delta$ Dificuldade_Bitcoin $n_{t-4}$ & 0.010 & 0.161 & 0.065 & 0.948 \\
\hline$\Delta$ Dificuldade_Bitcoin $n_{t-5}$ & -0.273 & 0.158 & -1.721 & 0.086051. \\
\hline$\Delta O$ ferta_Bitcoin $_{t-1}$ & -0.278 & 1.266 & -0.219 & 0.826 \\
\hline$\Delta O$ ferta_Bitcoin $_{t-2}$ & -3.327 & 1.352 & -2.460 & $0.014287 *$ \\
\hline$\Delta O$ ferta_Bitcoin $_{t-3}$ & 0.042 & 1.387 & 0.030 & 0.976 \\
\hline$\Delta O$ ferta_Bitcoin $_{t-4}$ & -1.631 & 1.483 & -1.100 & 0.272 \\
\hline$\Delta O$ ferta_Bitcoin $_{t-5}$ & 1.763 & 1.455 & 1.212 & 0.226 \\
\hline$\Delta$ Receita_Bitcoin ${ }_{t-1}$ & 0.255 & 0.055 & 4.661 & $4.24 \mathrm{e}-06^{* * *}$ \\
\hline$\Delta$ Receita_Bitcoin ${ }_{t-2}$ & 0.242 & 0.065 & 3.748 & $0.000203 * * *$ \\
\hline$\Delta$ Receita_Bitcoin ${ }_{t-3}$ & 0.087 & 0.065 & 1.339 & 0.181 \\
\hline$\Delta$ Receita_Bitcoin ${ }_{t-4}$ & -0.099 & 0.063 & -1.569 & 0.117 \\
\hline$\Delta$ Receita_Bitcoin $_{t-5}$ & -0.224 & 0.053 & -4.249 & $2.65 \mathrm{e}-05^{* * *}$ \\
\hline$\Delta$ Custo_Bitcoin $n_{t-1}$ & -0.167 & 0.047 & -3.530 & $0.000462 * * *$ \\
\hline$\Delta$ Custo_Bitcoin ${ }_{t-2}$ & -0.175 & 0.055 & -3.172 & $0.001628 * *$ \\
\hline$\Delta$ Custo_Bitcoin ${ }_{t-3}$ & 0.018 & 0.056 & 0.315 & 0.753 \\
\hline$\Delta$ Custo_Bitcoin ${ }_{t-4}$ & 0.107 & 0.058 & 1.856 & 0.064220 \\
\hline$\Delta$ Custo_Bitcoin ${ }_{t-5}$ & 0.157 & 0.059 & 2.649 & $0.008384 * *$ \\
\hline
\end{tabular}




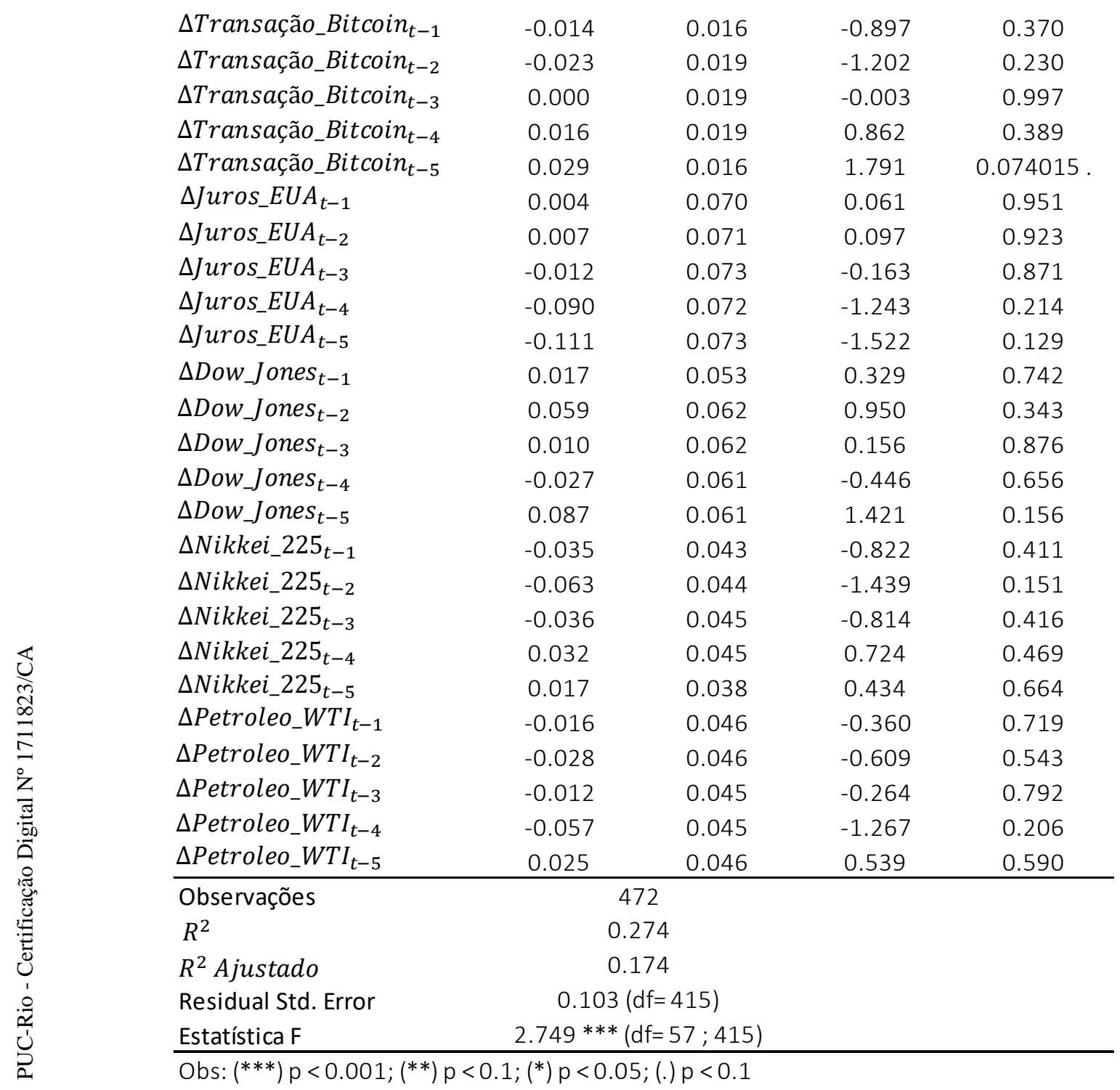

Tabela 8. Estimação VECM

Observando os resultados da estimação pelo VECM, podemos agrupar os resultados conforme a especificação do modelo apresentado no capítulo 3. Quanto aos componentes relacionados às forças de mercado, observamos que as quatro variáveis propostas possuem pelo menos uma de suas defasagens significativas. A oferta de Bitcoin é significante em sua segunda defasagem, a $5 \%$, e o número de transações de Bitcoin é significativo em sua quinta defasagem, a 10\%. Quanto às variáveis receita e custo do Bitcoin, há significância em pelo menos três de suas defasagens. Com relação aos impactos dessas variáveis, um aumento na oferta de Bitcoin sugere uma redução no preço do Bitcoin, enquanto o aumento do número 
de transações tem um efeito oposto sobre o preço. Um aumento nas receitas auferidas com Bitcoin possui um impacto positivo sobre o preço em suas primeiras defasagens, mas, ao longo das defasagens, esse efeito tende a diminuir o preço da criptomoeda. Diametralmente oposto, o aumento dos custos do Bitcoin tende a diminuir o preço da série do Bitcoin, mas, ao longo de suas defasagens, esse impacto se torna positivo.

Quanto ao impacto do reconhecimento público, não foi detectado nenhum coeficiente significativo, contrariando os achados de Kristoufek (2013). Esse efeito pode estar relacionado à periodização da série, observando que, atualmente, o conhecimento sobre essa criptomoeda é altamente difundido, diferentemente dos anos iniciais do Bitcoin, onde o conhecimento público poderia altamente influenciar seu preço.

Com relação ao impacto do fator tecnológico, observou-se que a dificuldade de mineração possui significância a $10 \%$ em sua quinta defasagem. O impacto desse coeficiente sobre o preço é negativo. Além disso, nenhum fator macroeconômico apresentou coeficientes significativos.

\section{4.}

Análise do Procedimento Autoregressivo de Defasagens Distribuídas

O segundo modelo aplicado consiste na estimação pelo procedimento autoregressivo de defasagens distribuídas (ARDL), o qual é amplamente utilizado na literatura para estimação de modelos que procuram entender os determinantes do preço do Bitcoin. Sua grande vantagem é permitir a estimação conjunta de séries que são estacionárias em níveis e são estacionárias em primeira diferença. Além disso, é apresentado um teste de bandas que testa a existência de relação de longo prazo entre as variáveis.

Os resultados da estimação pelo modelo ARDL são apresentados na Tabela 9. Com relação ao teste de bandas para a identificação de relação de longo prazo entre as variáveis, a estatística $F$ encontrada foi 6.140 , indicando a não rejeição da hipótese de existência de relação de longo prazo a 5\%. 


\begin{tabular}{|c|c|c|c|c|}
\hline & Coeficiente & Desvio Padrão & t valor & $\operatorname{Pr}(>|t|)$ \\
\hline Intercepto $(\alpha)$ & 0.064 & 0.034 & 1.859 & 0.063704 \\
\hline$\Delta$ Preço_Bitcoin ${ }_{t-1}$ & 0.674 & 0.052 & 12.873 & $<2 \mathrm{e}-16 * * *$ \\
\hline$\Delta$ Preço_Bitcoin $t_{t-2}$ & 0.215 & 0.061 & 3.513 & $0.000495^{* * *}$ \\
\hline$\Delta$ Preço_Bitcoin ${ }_{t-3}$ & -0.104 & 0.062 & -1.692 & 0.091400 \\
\hline$\Delta$ Preço_Bitcoin ${ }_{t-4}$ & 0.052 & 0.062 & 0.837 & 0.403 \\
\hline$\Delta$ Preço_Bitcoin $_{t-5}$ & -0.125 & 0.062 & -2.018 & $0.044227 *$ \\
\hline$\Delta$ Preço_Bitcoin ${ }_{t-6}$ & 0.103 & 0.051 & 2.026 & $0.043407 *$ \\
\hline Buscas_Google $_{t}$ & -0.013 & 0.010 & -1.278 & 0.202 \\
\hline$\Delta$ Buscas_Google $_{t-1}$ & 0.012 & 0.013 & 0.878 & 0.380 \\
\hline$\Delta$ Buscas_Google ${ }_{t-2}$ & -0.011 & 0.013 & -0.805 & 0.421 \\
\hline$\Delta$ Buscas_Google $e_{t-3}$ & 0.003 & 0.013 & 0.215 & 0.830 \\
\hline$\Delta$ Buscas_Google $e_{t-4}$ & -0.011 & 0.013 & -0.847 & 0.398 \\
\hline$\Delta$ Buscas_Google $e_{t-5}$ & 0.006 & 0.013 & 0.451 & 0.652 \\
\hline$\Delta$ Buscas_Google $t_{t-6}$ & 0.007 & 0.010 & 0.654 & 0.514 \\
\hline Dificuldade_Biticoin ${ }_{t}$ & 0.304 & 0.140 & 2.163 & $0.031112 *$ \\
\hline$\Delta$ Dificuldade_Bitcoin $n_{t-1}$ & -0.224 & 0.207 & -1.082 & 0.280 \\
\hline$\Delta$ Dificuldade_Bitcoin ${ }_{t-2}$ & 0.003 & 0.206 & 0.012 & 0.990 \\
\hline$\Delta$ Dificuldade_Bitcoin ${ }_{t-3}$ & -0.119 & 0.204 & -0.584 & 0.559 \\
\hline$\Delta$ Dificuldade_Bitcoin ${ }_{t-4}$ & 0.085 & 0.206 & 0.414 & 0.679 \\
\hline$\Delta$ Dificuldade_Bitcoin ${ }_{t-5}$ & -0.149 & 0.206 & -0.724 & 0.470 \\
\hline$\Delta$ Dificuldade_Bitcoin ${ }_{t-6}$ & 0.033 & 0.142 & 0.232 & 0.817 \\
\hline Oferta_Bitcoin ${ }_{t}$ & -1.133 & 1.184 & -0.957 & 0.339 \\
\hline$\Delta O$ ferta_Bitcoin $_{t-1}$ & 0.373 & 1.321 & 0.282 & 0.778 \\
\hline$\Delta O$ ferta_Bitcoin $_{t-2}$ & -2.730 & 1.243 & -2.196 & $0.028673 *$ \\
\hline$\Delta O$ ferta_Bitcoin $_{t-3}$ & 1.958 & 1.190 & 1.646 & 0.101 \\
\hline$\Delta O$ ferta_Bitcoin $_{t-4}$ & -0.945 & 1.217 & -0.777 & 0.438 \\
\hline$\Delta O$ ferta_Bitcoin $_{t-5}$ & 2.984 & 1.269 & 2.351 & $0.019196 *$ \\
\hline$\Delta O$ ferta_Bitcoin $_{t-6}$ & -0.397 & 1.173 & -0.338 & 0.735 \\
\hline Receita_Bitcoin ${ }_{t}$ & 0.297 & 0.048 & 6.245 & $1.09 \mathrm{e}-09 * * *$ \\
\hline$\Delta$ Receita_Bitcoin ${ }_{t-1}$ & 0.136 & 0.051 & 2.666 & $0.007982 * *$ \\
\hline$\Delta$ Receita_Bitcoin ${ }_{t-2}$ & -0.065 & 0.052 & -1.248 & 0.213 \\
\hline$\Delta$ Receita_Bitcoin ${ }_{t-3}$ & -0.154 & 0.050 & -3.050 & $0.002441 * *$ \\
\hline$\Delta$ Receita_Bitcoin ${ }_{t-4}$ & -0.177 & 0.051 & -3.482 & $0.000553 * * *$ \\
\hline$\Delta$ Receita_Bitcoin ${ }_{t-5}$ & -0.138 & 0.050 & -2.774 & $0.005805^{* *}$ \\
\hline$\Delta$ Receita_Bitcoin ${ }_{t-6}$ & 0.173 & 0.048 & 3.576 & $0.000393 * * *$ \\
\hline Custo_Bitcoin $_{t}$ & 0.053 & 0.042 & 1.282 & 0.200 \\
\hline$\Delta$ Custo_Bitcoin ${ }_{t-1}$ & -0.145 & 0.044 & -3.277 & $0.001141 * *$ \\
\hline$\Delta$ Custo_Bitcoin ${ }_{t-2}$ & -0.006 & 0.046 & -0.137 & 0.891 \\
\hline$\Delta$ Custo_Bitcoin ${ }_{t-3}$ & 0.167 & 0.045 & 3.721 & $0.000227^{* * *}$ \\
\hline$\Delta$ Custo_Bitcoin ${ }_{t-4}$ & 0.073 & 0.046 & 1.604 & 0.109 \\
\hline$\Delta$ Custo_Bitcoin ${ }_{t-5}$ & 0.054 & 0.045 & 1.211 & 0.227 \\
\hline$\Delta$ Custo_Bitcoin ${ }_{t-6}$ & -0.127 & 0.042 & -2.999 & $0.002877^{* *}$ \\
\hline
\end{tabular}




\begin{tabular}{|c|c|c|c|c|c|}
\hline & Transação_Bitcoin $_{t}$ & -0.027 & 0.014 & -1.888 & 0.059782. \\
\hline & $\Delta$ Transação_Bitcoin ${ }_{t-1}$ & 0.000 & 0.015 & -0.026 & 0.979 \\
\hline & $\Delta$ Transação_Bitcoin ${ }_{t-2}$ & 0.005 & 0.015 & 0.369 & 0.712 \\
\hline & $\Delta$ Transação_Bitcoin ${ }_{t-3}$ & 0.029 & 0.014 & 2.044 & $0.041583 *$ \\
\hline & $\Delta$ Transação_Bitcoin ${ }_{t-4}$ & 0.023 & 0.015 & 1.536 & 0.125 \\
\hline & $\Delta$ Transação_Bitcoin ${ }_{t-5}$ & 0.020 & 0.015 & 1.343 & 0.180 \\
\hline & $\Delta$ Transação_Bitcoin ${ }_{t-6}$ & -0.025 & 0.014 & -1.746 & 0.081508 \\
\hline & $J u r o s_{-} E U A_{t}$ & 0.034 & 0.062 & 0.553 & 0.581 \\
\hline & $\Delta J u r o s_{-} E U A_{t-1}$ & -0.029 & 0.076 & -0.384 & 0.701 \\
\hline & $\Delta J u r o s_{-} E U A_{t-2}$ & -0.038 & 0.078 & -0.487 & 0.627 \\
\hline & $\Delta J u r o s \_E U A_{t-3}$ & 0.050 & 0.080 & 0.621 & 0.535 \\
\hline & $\Delta J u r o s_{-} E U A_{t-4}$ & -0.051 & 0.079 & -0.645 & 0.519 \\
\hline & $\Delta J$ uros_EUA $A_{t-5}$ & -0.083 & 0.079 & -1.057 & 0.291 \\
\hline & $\Delta J u r o s_{-} E U A_{t-6}$ & 0.091 & 0.064 & 1.414 & 0.158 \\
\hline & Dow_Jones $_{t}$ & 0.111 & 0.047 & 2.359 & $0.018807 *$ \\
\hline & $\Delta$ Dow_Jones $_{t-1}$ & -0.114 & 0.066 & -1.735 & 0.083515 \\
\hline & $\Delta$ Dow_Jones $_{t-2}$ & 0.099 & 0.070 & 1.415 & 0.158 \\
\hline & $\Delta$ Dow_Jones $_{t-3}$ & -0.050 & 0.069 & -0.722 & 0.471 \\
\hline & $\Delta$ Dow_Jones $_{t-4}$ & -0.057 & 0.069 & -0.825 & 0.410 \\
\hline & $\Delta$ Dow_Jones $_{t-5}$ & 0.092 & 0.068 & 1.341 & 0.181 \\
\hline & $\Delta D_{o w_{-} \text {Jones }_{t-6}}$ & -0.065 & 0.054 & -1.217 & 0.224 \\
\hline & Nikkei_225 & 0.030 & 0.037 & 0.798 & 0.426 \\
\hline & $\Delta N i k k e i \_225_{t-1}$ & -0.093 & 0.048 & -1.938 & 0.053346 . \\
\hline$\underline{\underline{E}}$ & $\Delta N i k k e i \_225_{t-2}$ & -0.008 & 0.049 & -0.164 & 0.870 \\
\hline o & $\Delta N i k k e i \_225_{t-3}$ & 0.031 & 0.049 & 0.623 & 0.534 \\
\hline 5 & $\Delta$ Nikkei_225t-4 & 0.057 & 0.049 & 1.154 & 0.249 \\
\hline & $\Delta N i k k e i \_225_{t-5}$ & 0.037 & 0.047 & 0.799 & 0.425 \\
\hline $\bar{\Xi}$ & $\Delta N i k k e i_{-} 225_{t-6}$ & -0.058 & 0.033 & -1.774 & 0.076760 \\
\hline & Petroleo_WTI $I_{t}$ & 0.025 & 0.040 & 0.630 & 0.529 \\
\hline & $\Delta$ Petroleo_WTI $I_{t-1}$ & -0.035 & 0.053 & -0.670 & 0.503 \\
\hline 2 & $\Delta$ Petroleo_WTI $I_{t-2}$ & 0.031 & 0.054 & 0.586 & 0.558 \\
\hline & $\Delta$ Petroleo_WTI $I_{t-3}$ & 0.032 & 0.053 & 0.607 & 0.544 \\
\hline & $\Delta$ Petroleo_WTI $I_{t-4}$ & -0.092 & 0.053 & -1.727 & 0.084990 \\
\hline & $\Delta$ Petroleo_WTI $I_{t-5}$ & 0.058 & 0.054 & 1.081 & 0.280 \\
\hline & $\Delta$ Petroleo_WTI $I_{t-6}$ & 0.006 & 0.040 & 0.156 & 0.876 \\
\hline & Observações & \multicolumn{2}{|c|}{478} & & \\
\hline & $R^{2}$ & \multicolumn{2}{|c|}{0.994} & & \\
\hline & $R^{2}$ Ajustado & \multicolumn{2}{|c|}{0.992} & & \\
\hline & Residual Std. Error & \multicolumn{2}{|c|}{$0.088(\mathrm{df}=395)$} & & \\
\hline & Estatística F & \multicolumn{2}{|c|}{$792.3^{* * *}(\mathrm{df}=76 ; 395)$} & & \\
\hline
\end{tabular}

Obs: $\left({ }^{* * *}\right) p<0.001 ;\left({ }^{* *}\right) p<0.1 ;\left({ }^{*}\right) p<0.05 ;() p<$.

Tabela 9. Estimação ARDL

Reforçando os resultados do modelo VECM, o fator reconhecimento público, medido pela variável buscas no Google, não apresentou nenhum coeficiente 
significativo. Isso somente indica que, para a modelagem apresentada e para o período estudado, o reconhecimento público não apresentou impactos sobre a série de preços do Bitcoin.

Com relação ao fator tecnológico, o coeficiente da dificuldade de mineração é significativo em nível a 5\%. A diferença comparada ao modelo VECM é com relação ao seu impacto: enquanto um aumento na dificuldade de mineração no modelo VECM tem um impacto negativo sobre os preços, no modelo ARDL, a dificuldade de mineração gera um impacto positivo sobre a série de preços do Bitcoin.

Das variáveis macroeconômicas incluídas no modelo, somente a taxa de juros americana não apresentou coeficientes significativos. O coeficiente da série em nível do Dow Jones apresentou um impacto positivo sobre os preços, enquanto sua primeira defasagem apresenta um impacto contrário. Quanto aos coeficientes da Nikkei e dos preços do petróleo, um aumento em seus preços impacta negativamente os preços do Bitcoin. Podemos observar, nesse caso, um possível tradeoff entre a alocação dos recursos em Bitcoin ou alocar os recursos em bolsas de valores ou em commodities.

Por fim, todas as variáveis que representam as forças de mercado apresentaram significância em pelo menos uma de suas defasagens. Os resultados do modelo ARDL seguem os resultados do modelo VECM, observando que o impacto dos coeficientes de cada uma das variáveis dependerá da defasagem.

Em suma, foram encontrados resultados significativos após a aplicação dos modelos VECM e ARDL, seguindo o que foi proposto nos objetivos do presente estudo. Somente o termo de reconhecimento público não apresentou coeficientes significativos, diferentemente dos resultados de Kristoufek (2013) e Lin e Wang (2016). O próximo capítulo apresentará a comparação dos resultados com a literatura e as considerações finais. 


\section{5 Conclusões}

O presente estudo se propôs a apresentar uma abordagem a qual seja possível identificar os determinantes do preço do Bitcoin, além de contribuir com o aumento da literatura sobre criptomoedas no Brasil, a qual é significantemente escassa.

O modelo especificado foi baseado na literatura existente sobre a determinação do preço do Bitcoin, definindo quatro grupos de variáveis que poderiam influenciar no movimento de preços da criptomoeda. São eles: fatores de mercado, fatores tecnológicos, fatores macroeconômicos e reconhecimento público. Esses modelos foram estimados a partir do modelo do vetor de correção de erros (VECM), utilizado em séries que são estacionárias em primeira diferença, e pelo procedimento autoregressivo de defasagens distribuídas (ARDL), o qual permite a estimação conjunta de séries que são estacionárias em nível e em primeira diferença.

Com relação ao impacto de fatores tecnológicos, apresentado de forma pioneira em Lin e Wang (2016), foi utilizada a dificuldade de mineração do Bitcoin. Nos resultados desse estudo, encontrou-se significância nos coeficientes desse termo em ambos os modelos, mas os impactos sobre a série de preços do Bitcoin apresentam efeitos opostos quando é comparado os modelos. Os resultados desses estudos corroboram com os achados de Lin e Wang (2016), nos quais esse termo também se apresentou significativo.

O termo reconhecimento público, dimensionado por uma proxy de quantidade de buscas no Google, não apresentou significância em nenhum dos modelos estudados. Os resultados são contrários aos achados de Kristoufek (2013), que apresenta em seu paper uma relação entre reconhecimento público e o preço do Bitcoin. Lin e Wang (2016) corroboram com os resultados de Kristoufek (2013). Uma possível explicação para a diferença dos resultados seria a periodização da série, observando que o reconhecimento sobre o tema Bitcoin foi amplamente difundido nos últimos anos, diferentemente do período da série que Kristoufek 
(2013) e Lin e Wang (2016) analisaram, os quais cobriram os anos iniciais do Bitcoin.

Com relação aos fatores econômicos, somente o modelo ARDL apresentou significância para seus componentes. Os resultados desses estudos são comparáveis aos resultados de Van Wijk (2013), que dimensionou o impacto de algumas bolsas de ações e de commodities sobre os preços do Bitcoin.

Os impactos das forças de mercado são apresentados em diversos estudos, como em Buchholtz et al. (2012), Bouoiyour e Selmi (2015) e Ciaian, Rajcaniova e Kancs (2016). É visto que a inclusão dessas variáveis é comumente utilizada nos estudos do preço do Bitcoin, e tais variáveis se apresentam sempre importantes para entender a formação de preços da criptomoeda. Nesse estudo, foram utilizadas as séries das receitas, custos, oferta e número de transações do Bitcoin a fim de entender se os resultados desse estudos corroboravam com a literatura. Foi encontrada significância nessas variáveis em ambos os modelos, VECM e ARDL, seguindo o que é apresentado por diversos autores.

As Tabelas 10 e 11 apresentam uma síntese dos resultados dos testes VECM e ARDL, além de seus respectivos impactos.

\begin{tabular}{|c|c|}
\hline $\begin{array}{l}\text { Força de Mercado: } \\
\text { Os quatro componentes possuem } \\
\text { pelo menos uma de suas } \\
\text { defasagens significativas. }\end{array}$ & 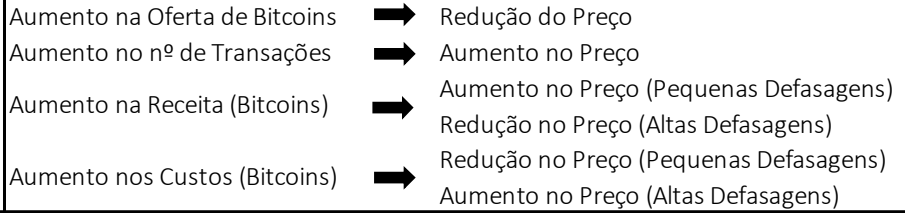 \\
\hline $\begin{array}{l}\text { Fatores Tecnológicos: } \\
\text { A dificuldade de mineração } \\
\text { apresenta pelo menos uma } \\
\text { defasagem significativa. }\end{array}$ & $\begin{array}{l}\text { Aumento na Dificuldade de } \\
\text { Mineração }\end{array}$ \\
\hline $\begin{array}{l}\text { Reconhecimento Público: } \\
\text { A variável Buscas_Google não } \\
\text { apresentou nenhuma variável } \\
\text { significativa. }\end{array}$ & $\begin{array}{l}\text { Pode estar relacionado com a periodização da série, observando que atualmente o } \\
\text { conhecimento sobre essa criptomoeda é altamente difundido, diferentemente dos } \\
\text { anos iniciais do Bitcoin, onde o conhecimento público poderia altamente influenciar } \\
\text { seu preço. }\end{array}$ \\
\hline Fatores Macroeconômicos: & $\begin{array}{l}\text { Nenhuma das variáveis do modelo apresentaram variáveis significativas para o } \\
\text { VECM. }\end{array}$ \\
\hline
\end{tabular}

Tabela 10. Impactos dos coeficiêntes no modelo VECM 


\begin{tabular}{|c|c|c|}
\hline $\begin{array}{l}\text { Força de Mercado: } \\
\text { Os quatro componentes possuem pelo } \\
\text { menos uma de suas defasagens } \\
\text { significativas, corroborando com o }\end{array}$ & $\begin{array}{l}\text { Aumento na Oferta de Bitcoins } \\
\text { Aumento no no de Transações } \\
\text { Aumento na Receita (Bitcoins) } \\
\text { Aumento nos Custos (Bitcoins) }\end{array}$ & $\begin{array}{l}\Rightarrow \text { Aumento (Pequenas Defasagens)/ Redução (Grandes Defasagens) } \\
\Rightarrow \text { Aumenta ou reduz, dependendo da defasagem } \\
\Rightarrow \text { Aumenta ou reduz, dependendo da defasagem } \\
\Rightarrow \text { Aumenta ou reduz, dependendo da defasagem }\end{array}$ \\
\hline $\begin{array}{c}\text { Fatores Tecnológicos: } \\
\text { A dificuldade de mineração apresenta } \\
\text { pelo menos uma defasagem } \\
\text { significativa. No entanto, o impacto da }\end{array}$ & $\begin{array}{l}\text { Aumento na Dificuldade de } \\
\text { Mineração }\end{array}$ & $\Rightarrow$ Aumento do Preço \\
\hline Reconhecimento Público: & A variável Buscas_Google não ap & $\begin{array}{l}\text { esentou nenhuma variável significativa para o modelo ARDL, reforçando } \\
\text { os resultados do modelo VECM. }\end{array}$ \\
\hline $\begin{array}{c}\text { Fatores Macroeconômicos: } \\
\text { Somente a taxa de juros americana não } \\
\text { apresentou significancia no modelo } \\
\text { ARDL. }\end{array}$ & $\begin{array}{l}\text { Aumento no índice Dow Jones } \\
\text { Aumento no índice Nikkei } \\
\text { Aumento no índice WTI }\end{array}$ & $\begin{aligned} & \text { Aumento no Preço (Pequenas Defasagens) } \\
& \text { Redução no Preço (Altas Defasagens) } \\
\Rightarrow & \text { Redução no Preço } \\
\Rightarrow & \text { Redução no Preço }\end{aligned}$ \\
\hline
\end{tabular}

Tabela 11. Impactos dos coeficiêntes no modelo ARDL

Por fim, é importante ressaltar que o estudo dos preços do Bitcoin ainda é recente e que ainda é possível acrescentar diversos fatores os que possam influenciar na flutuação de preço da criptomoeda. No entanto, os fatores apresentados no escopo desse estudo são importantes para entender o comportamento de uma criptomoeda, visto a grande volatilidade em seus preços. 
6

Referências Bibliográficas

ALBUQUERQUE, B. S.; CALLADO, M. C. Understanding Bitcoins: Facts and Questions. In: Revista Brasileira de Economia, vol. 69, n. 1, p. 03-16, mar. 2015.

BOUOIYOUR, J., SELMI, R.. What Does BitCoin Look Like? MPRA Paper No. 58091, 2015. Germany: University Library of Munich.

BUCHHOLZ, M., DELANEY J., WARREN J., PARKER J. Bits and Bets, Information, Price Volatility, and Demand for BitCoin, Economics 312. Disponível em: <http://www.bitcointrading.com/pdf/bitsandbets.pdf>, 2012. CARVALHO, F. J. C. SOUZA, F. E. P. Economia Monetária e Financeira. $3^{\mathrm{a}}$ ed. Rio de Janeiro: Elsevier, 2015.

CIAIAN, P., RAJCANIOVA, M., KANCS, D. The economics of Bitcoin price formation. Applied Economics, 2016, volume 48, número 19, p. 17991815.

CROSBY, M., NACHIAPPAN, PATTANAYAK, P., VERMA, S., KALYANARAMAN, V. Blockchain technology: beyond Bitcoin. Sutardja Center for Entrepreneurship \& Tecnology, Berkeley Engineering, 2015.

DAl, W. Wei Dai. B-money, 1998. Disponível em: <http://www.weidai.com/bmoney.txt>. Acesso em: 15 dezembro 2018.

DWYER, G. P. The economics of Bitcoin and similar private digital currencies. Journal of Financional Stability, v. 17, pp. 81-91, 2015.

FARR, C. Bitcoin Regulation, Here We Come: Singapore Clarifies Tax Polices. Venture Beat, 10 jan. 2014. Disponível em: $<$ https://venturebeat.com/2014/01/10/singapore-clarifies-tax-on-bitcoinexchanges-and-sales> Acesso em: 20 out. 2018 .

HAMILTON, J. D. Time series analysis. Princeton University Press, 1994. JOHANSEN, S. Statistical analysis of cointegration vectors. Journal of Economic Dynamics and Control, Boston, v. 12, p. 231-54, 1988. 
KRISTOUFEK, L. Bitcoin Meets Google Trends and Wikipedia: Quantifying the Relationship between Phenomena of the Internet Era. Scientific Reports 3 (3415): 1-7. doi:10.1038/srep03415, 2013.

$\mathrm{LI}, \mathrm{X}$., WANG, C. The tecnology and economic determinants of criptocurrency exchange rates: The case of Bitcoin. Elsevier, 2016. Disponível em: http://dx.doi.org/10.1016/j.dss.2016.12.001.

NAKAMOTO, S. Bitcoin: A Peer-to-Peer Electronic Cash System. In: Bitcoin Org, 2008. Disponível em: <https://bitcoin.org/bitcoin.pdf>. Acesso em: 14 outubro 2018.

PANTULA, S. G. Testing for Unit Roots in Time Series Data. Econometric Theory, v. 5, n. 2, p.256-271,1989.

PESARAN, H.; SHIN, Y. An autoregressive distributed lag modelling approach to cointegration analysis. In: Econometrics and Economic Theory in the 20th Century: The Ragnar Frisch Centennial Symposium, Strom S (ed.). Cambridge University Press: Cambridge, 1999.

PESARAN, H.; SHIN, Y.; SMITH, R. J. Bounds testing approaches to the analysis of level relationships, Journal of Applied Econometrics, n. 16. V. 3, p. 289-326, 2001.

SWAN, M. Blockchain: Blueprint for a new economy. O'Reilly Media Inc. 2015.

TAPSCOTT, D., TAPSCOTT, A. Blockchain Revolution: como a tecnologia por trás do Bitcoin está mudando o dinheiro, os negócios e o mundo. SENAI-SP Editora, 2016.

ULRICH, F. Bitcoin: a moeda na era digital. Instituto Ludwig von Mises Brasil, 2014.

VAN WIJK, D. What can be expected from the BitCoin? Working Paper No. 345986. Rotterdam: Erasmus Rotterdam Universiteit, 2013.

YERMACK, D. Is Bitcoin a real currency? An economic appraisal. National Bureau of Economic Research, 2013.

ZIVOT, E.; WANG, J. Modeling financial time series with S-plus. 2. ed. New York: Springer, 2005. 